\title{
Analysis of Food Habits during Pandemic in a Polish Population-Based Sample of Primary School Adolescents: Diet and Activity of Youth during COVID-19 (DAY-19) Study
}

\author{
Aleksandra Kołota *(D) and Dominika Głąbska (D) \\ Department of Dietetics, Institute of Human Nutrition Sciences, \\ Warsaw University of Life Sciences (SGGW-WULS), 159c Nowoursynowska Street, \\ 02-776 Warsaw, Poland; dominika_glabska@sggw.edu.pl \\ * Correspondence: aleksandra_kolota@sggw.edu.pl; Tel.: +48-22-5937186
}

check for updates

Citation: Kołota, A.; Głąbska, D. Analysis of Food Habits during Pandemic in a Polish

Population-Based Sample of Primary School Adolescents: Diet and Activity of Youth during COVID-19 (DAY-19) Study. Nutrients 2021, 13, 3711. https://doi.org/10.3390/nu13113711

Academic Editors: Carlo Agostoni and Gregorio Paolo Milani

Received: 7 September 2021

Accepted: 19 October 2021

Published: 22 October 2021

Publisher's Note: MDPI stays neutral with regard to jurisdictional claims in published maps and institutional affiliations.

Copyright: (c) 2021 by the authors. Licensee MDPI, Basel, Switzerland. This article is an open access article distributed under the terms and conditions of the Creative Commons Attribution (CC BY) license (https:/ / creativecommons.org/licenses/by/ $4.0 /)$.
Abstract: The improper dietary behaviors of children and adolescents during the COVID-19 pandemic, which are associated with lockdowns and reduced physical activity, are a complex problem, potentially resulting in increased risk of diet-related diseases, including overweight and obesity and their consequences. The aim of the study was to assess the food habits during the COVID-19 pandemic and to define their association with physical activity and body mass changes in a Polish population of primary school adolescents within the Diet and Activity of Youth During COVID-19 (DAY-19) Study. The DAY-19 Study was conducted in June 2020 in a national cohort of 1334 primary school students aged 10-16 years, recruited based on a stratified random sampling of schools (sampling counties from voivodeships and schools from counties). The Adolescent Food Habits Checklist (AFHC) was used to assess food habits, associated with food purchase, preparation, and consumption, which in the studied group were analyzed separately for the period before (retrospective data) and during the COVID-19 pandemic (prospective data). The recognizable physical activity changes and recognizable body mass changes were also assessed (retrospective data) and respondents were classified as those declaring that their physical activity and body mass decreased, remained stable, or increased during the COVID-19 pandemic. It was observed that during the COVID-19 pandemic the majority of food habits changed in a statistically significant way $(p<0.05)$. Within food purchase habits, the number of respondents who declared choosing a low-fat lunch away from home decreased, often buying pastries or cakes decreased, and buying a low-fat crisps brand increased $(p<0.05)$. Within food preparation habits, the number of respondents who declared trying to keep overall fat intake down increased, trying to keep overall sugar intake down increased, eating at least one serving of vegetables or salad with evening meal increased, and usually including some chocolate and/or biscuits in a packed lunch decreased $(p<0.05)$. Within food consumption habits, the number of respondents who declared making sure that they eat at least one serving of fruit a day increased, eating at least three servings of fruit most days increased, making sure that they eat at least one serving of vegetables or salad a day increased, trying to ensure that they eat plenty of fruit and vegetables increased, often choosing a fruit when they have a snack between meals increased, eating at least three servings of fruit most days increased, and generally trying to have a healthy diet increased $(p<0.05)$. It was concluded that in the period of the COVID-19 pandemic and resultant remote education, adolescents in Poland presented different food habits than before, while the majority of changes were positive. The positive food purchase, preparation, and consumption habits were observed mainly in sub-groups of adolescents declaring decreased body mass or increased physical activity during the COVID-19 pandemic. It may be suggested that physical activity may support positive changes of dietary behaviors and while combined positive changes of diet and increased physical activity, they may effectively promote body mass reduction in adolescents. 
Keywords: adolescents; body mass; obesity; diet; physical activity; food habits; Adolescents' Food Habits Checklist (AFHC); COVID-19 pandemic; DAY-19 study

\section{Introduction}

The puberty period is crucial for the development of organisms due to key changes of anatomy, physiology, neurology, and behavior [1]. In this period, food preferences and food habits are created and strengthened, while changing from those characteristics for childhood to those stable through adulthood [2]. As a result, influencing food habits in the period of adolescence is a unique possibility to reduce the risk of diet-related health problems in adulthood [3]. A diversified and balanced diet enables proper development and growth of an organism, increased physical and mental functioning, and maintaining a proper body mass $[4,5]$.

Especially important are fruit and vegetables, being directly associated with decreased risk of non-communicable diseases, including obesity [6,7], but also cardiovascular diseases [8], diabetes [9], and cancer in adulthood [10,11]. Moreover, fruit and vegetables' intake in adolescence influences mental health [12] and self-assessment of health, including mental health [13]. As a result, the World Health Organization (WHO) emphasizes that a lot of countries recommended for adolescents to consume at least $400 \mathrm{~g}$ of fruit and vegetables per day [3], but a number of adolescents do not meet this recommendation [14] and in some countries their intake is decreasing, as was stated for fruit consumption in Germany, Greenland, Greece, Poland, and Portugal, as well as for vegetable consumption in Germany, Lithuania, Latvia, Poland, and Russia [15]. The indicated improper dietary habits in adolescents are not only a predictor of improper dietary habits in adults [16], but also are associated with excessive body mass [17], while following dietary recommendations protects from this [18].

The current situation of the outbreak of Coronavirus Disease 2019 (COVID-19) pandemic, which was associated with lockdowns and social isolation in numerous studies, also caused changes of nutritional behaviors, including shopping behaviors, that were observed both for adults $[19,20]$, children, and adolescents [21,22]. However, the results of the studies conducted so far are not congruent, as, depending on the studied population, the research conducted so far revealed either positive or negative changes of eating behaviors. A study conducted in Spain indicated negative changes, including a decrease of fruit and vegetables' intake in children and adolescents [21]. A study conducted in Brazil indicated positive changes, including reduced intake of unhealthy processed food products by families during their isolation [23]. At the same time, another study conducted in several countries including Brazil (Italy, Spain, Chile, Colombia, and Brazil) indicated positive changes resulting from more time spent by families on home cooking, but for adolescents no positive changes were observed [24].

Simultaneously, the COVID-19 pandemic reduced for children and adolescents the possibilities of physical activity and team sports' practicing, due to remote learning systems as well as closed playgrounds and courts, which caused, in a number of studies, a reduced physical activity level in this population group [25-28]. However, in a number of studies conducted in a population of adolescents, while sport activities were decreased, habitual physical activities and outdoor activities, such as gardening, housework, cycling, skiing, or walking, were increased $[29,30]$. Such a situation results from the shift of physical activity from team sports and indoor sport activities to individual sports and outdoor sport activities, being imposed by legal regulations associated with the COVID-19 pandemic [31], but such an approach sometimes requires the commitment of parents [32]. As reduced physical activity in children and adolescents is commonly associated with worse nutritional habits $[33,34]$, it should be indicated as a complex problem, potentially resulting in increased risk of diet-related diseases [35], including overweight and obesity [36]. Taking this 
into account, the food habits of children and adolescents during the COVID-19 pandemic are becoming a serious public health issue to be addressed.

Among various tools used to assess food habits, one of the commonly used questionnaires is the Adolescent Food Habits Checklist (AFHC) by Johnson et al. [37], which is used not only for adolescents [38,39] but also for adults [40]. This questionnaire was already used to assess the changes of food habits during the COVID-19 pandemic in the population of older adolescents [41,42], and studies revealed that while some food habits were improved, the other ones were worsened, which enabled defining essential areas for public health education [41]. In populations of younger adolescents [37,38], or even children [39], this tool was also used in multiple studies, but it has not been used so far in this population during the COVID-19 pandemic to assess their food habits changes.

Taking into consideration the issues described above, the aim of this study was to assess food habits during the COVID-19 pandemic and to define their association with physical activity and body mass changes in a Polish population of primary school adolescents within the Diet and Activity of Youth During COVID-19 (DAY-19) Study.

\section{Materials and Methods}

\subsection{Ethical Statement}

The conducted study was approved by the Ethics Committee of the Institute of Human Nutrition Sciences of the Warsaw University of Life Sciences (no. 18/2020). Within the study, the diet and physical activity were assessed [43] and their associations with body mass changes were analyzed.

All the procedures were based on the recommendations of the Declaration of Helsinki. From the participants of the study and their parents/legal guardians, their informed consent for the study participation was obtained.

\subsection{The Population Studied within DAY-19 Study}

The DAY-19 Study was conducted in a Polish national cohort of primary school students aged 10-16 years. The participants of the study were recruited in June 2020, based on a stratified random sampling of schools, which was conducted in two phases, including: (1) sampling of counties from voivodeships (basic administrative units of Poland) and (2) sampling of schools from counties, which was conducted while using the Polish register of primary schools. The procedure was in agreement with the general approach applied in Poland to obtain a representative sample of students from all regions of the country, chosen especially during the COVID-19 pandemic [44,45]. In the first phase, 10 counties were sampled from each voivodeship ( 10 counties $\times 16$ voivodeships) and in the second phase, 10 primary schools were sampled from each county (10 schools $\times 160$ counties). All the sampled schools were invited to participate in the study, while the principal of each of them received a written invitation, accompanied with detailed protocol and all the information. If principals expressed the will for the school to participate in the study, they informed students that they were invited to participate, while their participation was also voluntary. The students were included only if they, as well as their parents/legal guardians, provided informed consent to participate. If so, they were provided an electronic link to the dedicated questionnaire which did not allow the identification of the study participants and did not gather any personal or sensitive data, except for age and the attended primary school.

All the students were encouraged to participate in the study, but similarly as for schools, a low response rate was observed. The participation in the study was voluntary both for schools and within the schools for students. The final number of participating schools was 43, and they represented all the regions of Poland.

The inclusion criteria for the participants of the study were as follows:

- Being a student of the sampled school;

- Being 10-16 years old;

- Informed consent for the study participation provided from both students and their parents/legal guardians. 
No exclusion criteria based on the health status of participants were formulated, which was associated with the fact that the aim of the study was to assess general food habits during the COVID-19 pandemic in a population of Polish adolescents, independently from the presence of diet-related diseases.

After collecting the completed questionnaires, some of them were excluded from the analysis, based on the following criteria:

- Any missing data in the provided questionnaire;

- Any unreliable data in the provided questionnaire (as in the other research [46], the data was considered to be unreliable if the respondent provided uniform answers across all of the questions, which was associated with failing to differentiate between answer choices, as they provided identical responses to all questions).

The final sample of 1334 students participated in the study. The recruitment procedure is presented on flow chart (Figure 1).

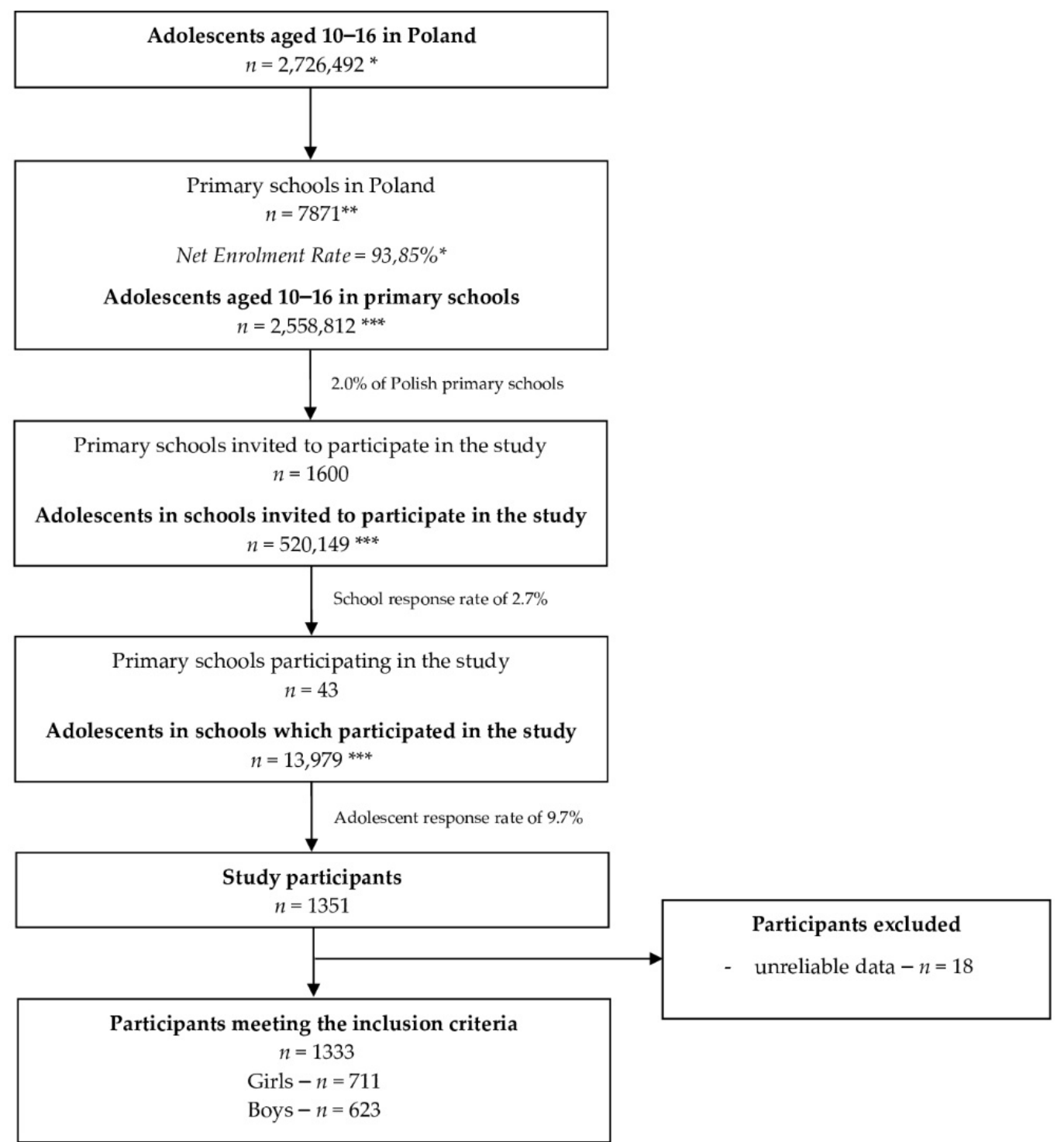

Figure 1. The recruitment procedure on flow chart for the adolescents from the Diet and Activity of Youth during COVID-19 (DAY-19) Study cohort $(n=1333){ }^{*}[47,48]^{* *}[49]^{* * *}$ calculated based on data of the Central Statistical Office. 


\subsection{Applied Questionnaire}

The applied questionnaire included basic questions about recognizable body mass changes during the COVID-19 pandemic and recognizable physical activity changes during the COVID-19 pandemic, as well as included the AFHC by Johnson et al. [37], which is validated in a population of adolescents [37]. As AFHC is a questionnaire developed to be completed by adolescents, all the information was self-reported.

In the period when the study was conducted, based on the decision of the Polish Ministry of National Education, in Poland the system of remote learning was implemented [50], so in order to reflect properly changes of food habits during the COVID-19 pandemic, participants of the study were asked to describe separately their food habits before the period of remote learning (being the period before the COVID-19 pandemic) and current food habits (in the period of remote learning). Similarly, as in the previous Polish studies conducted in the population of adolescents [41], the question was formulated based on ensuring the period before implementing remote learning and the period of remote learning were easily recognizable for adolescents, as for adolescents the period of COVID-19 pandemic itself may be not so easy to define.

The AFHC [37] is based on 23 questions about various food habits, which are associated with food purchase, preparation, and consumption. The Polish version of AFHC was applied as it is commonly used [41,42], and was developed based on the forward and backward translation and transcultural adaptation [41].

The question about recognizable physical activity changes during the COVID-19 pandemic was based on the subjective assessment of participants. They were asked to declare if their physical activity decreased, remained stable, or increased (simple one-choice question to choose one option: decreased, remained stable, or increased). At the same time, the question about recognizable body mass changes during the COVID-19 pandemic was supported by the information about the current height and weight of participants, as well as their height and weight before the period of remote learning. The participants were asked about their current weight, and afterwards they were asked to within a simple onechoice question choose one option: that it decreased, remained stable, or increased during the COVID-19 pandemic. If they declared that their weight increased or decreased, they were asked to declare what was their weight before the COVID-19 pandemic. The Body Mass Index (BMI) values were calculated for each participant using a standard Quetelet equation [51] and they were assessed using the Polish growth reference values, based on gender and age [52], with dedicated software [53]. As for physical activity, respondents were classified as those whose body mass decreased, remained stable, or increased during the COVID-19 pandemic. In order to obtain reliable answers, respondents were informed that their answers will not be judged in any way and that they are needed only for the scientific research.

\subsection{Statistical Analysis}

The statistical analysis was based on the comparison of the frequency of the specific answers provided by respondents for the periods before and during the COVID-19 pandemic, assessed based on identical questions formulated twice (separately for the indicated periods), while the AFHC was used. The assessment included assessment of the following factors:

- Period of the COVID-19 pandemic (comparison of the answers provided for the period before and during the COVID-19 pandemic);

- Physical activity changes (comparison of the answers provided by respondents declaring that their physical activity decreased, remained stable, or increased during the COVID-19 pandemic);

- Body mass changes (comparison of the answers provided by respondents declaring that their body mass decreased, remained stable, or increased during the COVID-19 pandemic). 
The statistical analysis was conducted while using the chi-square test (for the comparison of the frequency of specific answers for the period before and during the COVID-19 pandemic) and McNemar's test (for the assessment of changes of answers provided by specific respondents in longitudinal analysis, while comparing period before and during the COVID-19 pandemic). The Statgraphics Plus for Windows 5.1 software (Statgraphics Technologies Inc., The Plains, VA, USA) was used. The values of $p \leq 0.05$ were interpreted as statistically significant differences.

\section{Results}

\subsection{Comparison of Results before and during the COVID-19 Pandemic}

The food purchase habits assessed while using AFHC in the period before the COVID19 pandemic and during the COVID-19 pandemic, as declared by the adolescents from the DAY-19 Study cohort, are presented in Table 1. During the COVID-19 pandemic, the number of respondents who declared choosing a low-fat lunch away from home decreased $(40.4 \%$ vs. $34.0 \%, p<0.0001)$. At the same time, the number of respondents who declared often buying pastries or cakes decreased $(33.6 \%$ vs. $24.1 \%, p<0.0001)$, but the number of respondents who declared buying a low-fat crisps brand increased $(22.6 \%$ vs. $24.8 \%$, $p<0.05)$. The longitudinal analysis confirmed the significant changes of the food purchase habits in the period of the COVID-19 pandemic, which was stated for all the habits except for choosing a low-fat option while having lunch away from home.

Table 1. The food purchase habits assessed while using Adolescents' Food Habits Checklist (AFHC) in the period before the COVID-19 pandemic and during the COVID-19 pandemic, as declared by the adolescents from the Diet and Activity of Youth during COVID-19 (DAY-19) Study cohort $(n=1333)$.

\begin{tabular}{|c|c|c|c|c|c|}
\hline \multicolumn{2}{|c|}{ Food Purchase Habits Assessed within AFHC * } & \multirow{2}{*}{$\begin{array}{c}\text { Before COVID-19 } \\
\text { Pandemic } \\
538(40.4 \%)\end{array}$} & \multirow{2}{*}{$\begin{array}{l}\text { During the COVID-19 } \\
\text { Pandemic } \\
453(34.0 \%)\end{array}$} & $p^{* *}$ & $p^{* * *}$ \\
\hline \multirow{3}{*}{$\begin{array}{l}\text { If I am having lunch away from home, I } \\
\text { often choose a low-fat option }\end{array}$} & True & & & \multirow{3}{*}{$<0.0001$} & \multirow{3}{*}{0.7344} \\
\hline & False & $392(29.4 \%)$ & $365(27.4 \%)$ & & \\
\hline & Not applicable & $403(30.2 \%)$ & $515(38.6 \%)$ & & \\
\hline \multirow{3}{*}{$\begin{array}{l}\text { If I am buying crisps, I often choose a } \\
\text { low-fat brand }\end{array}$} & True & $301(22.6 \%)$ & $331(24.8 \%)$ & \multirow{3}{*}{0.0139} & \multirow{3}{*}{$<0.0001$} \\
\hline & False & $799(59.9 \%)$ & $726(54.5 \%)$ & & \\
\hline & Not applicable & $233(17.5 \%)$ & $276(20.7 \%)$ & & \\
\hline \multirow{2}{*}{ I often buy pastries or cakes } & True & $448(33.6 \%)$ & $322(24.1 \%)$ & \multirow{2}{*}{$<0.0001$} & \multirow{2}{*}{$<0.0001$} \\
\hline & False & $885(66.4 \%)$ & $1011(75.8 \%)$ & & \\
\hline \multirow{2}{*}{ I rarely eat takeaway meals } & True & $1085(81.4 \%)$ & $1108(83.1 \%)$ & \multirow{2}{*}{0.2435} & \multirow{2}{*}{$<0.0001$} \\
\hline & False & $248(18.6 \%)$ & $225(16.9 \%)$ & & \\
\hline \multirow{2}{*}{$\begin{array}{l}\text { When I am buying a soft drink, I } \\
\text { usually choose a diet drink }\end{array}$} & True & $481(36.1 \%)$ & $530(39.8 \%)$ & \multirow{2}{*}{0.0505} & \multirow{2}{*}{0.0001} \\
\hline & False & $852(63.9 \%)$ & $803(60.2 \%)$ & & \\
\hline \multirow{3}{*}{$\begin{array}{l}\text { If I am having a dessert or pudding in a } \\
\text { restaurant, I usually choose the } \\
\text { healthiest one }\end{array}$} & True & $214(16.5 \%)$ & $226(16.9 \%)$ & \multirow{3}{*}{0.2732} & \multirow{3}{*}{$<0.0001$} \\
\hline & False & $576(43.2 \%)$ & $535(40.2 \%)$ & & \\
\hline & Not applicable & $543(40.7 \%)$ & $572(42.9 \%)$ & & \\
\hline
\end{tabular}

*AFHC-Adolescents' Food Habits Checklist [37]; ** chi-square test (for the comparison of the frequency of specific answers); ${ }^{* * *}$ McNemar's test (for longitudinal analysis).

The food preparation habits assessed while using AFHC in the period before the COVID-19 pandemic and during the COVID-19 pandemic, as declared by the adolescents from the DAY-19 Study cohort, are presented in Table 2. During the COVID-19 pandemic, the number of respondents who declared trying to keep overall fat intake down increased $(54.3 \%$ vs. $60.0 \%, p<0.01)$ and the number of respondents who declared trying to keep overall sugar intake down also increased $(55.6 \%$ vs. $61.7 \%, p<0.01)$. At the same time, the number of respondents who declared eating at least one serving of vegetables or salad with evening meal increased $(75.5 \%$ vs. $79.8 \%, p<0.01)$, while the number of respondents who declared usually including some chocolate and/or biscuits in a packed lunch decreased (32.6\% vs. $23.1 \%, p<0.0001)$. The longitudinal analysis confirmed the significant changes 
of the food preparation habits in the period of the COVID-19 pandemic, which was stated for all the habits except for eating at least one serving of vegetables with the evening meal.

Table 2. The food preparation habits assessed while using Adolescents' Food Habits Checklist (AFHC) in the period before the COVID-19 pandemic and during the COVID-19 pandemic, as declared by the adolescents from the Diet and Activity of Youth during COVID-19 (DAY-19) Study cohort $(n=1333)$.

\begin{tabular}{|c|c|c|c|c|c|}
\hline \multicolumn{2}{|c|}{ Food Preparation Habits Assessed within AFHC * } & \multirow{3}{*}{$\begin{array}{c}\begin{array}{c}\text { Before COVID-19 } \\
\text { Pandemic }\end{array} \\
509(38.2 \%) \\
824(61.8 \%)\end{array}$} & \multirow{3}{*}{$\begin{array}{c}\begin{array}{c}\text { During COVID-19 } \\
\text { Pandemic }\end{array} \\
536(40.2 \%) \\
797(59.8 \%)\end{array}$} & \multirow{3}{*}{$\begin{array}{c}p^{* *} \\
0.2842\end{array}$} & \multirow{3}{*}{$\begin{array}{l}p^{* * *} \\
<0.0001\end{array}$} \\
\hline \multirow{2}{*}{ I usually avoid eating fried foods } & True & & & & \\
\hline & False & & & & \\
\hline \multirow{2}{*}{ I try to keep my overall fat intake down } & True & $724(54.3 \%)$ & $800(60.0 \%)$ & \multirow{2}{*}{0.0029} & \multirow{2}{*}{$<0.0001$} \\
\hline & False & $609(45.7 \%)$ & $533(40.0 \%)$ & & \\
\hline \multirow{2}{*}{$\begin{array}{l}\text { I try to keep my overall sugar } \\
\text { intake down }\end{array}$} & True & $741(55.6 \%)$ & $822(61.7 \%)$ & \multirow{2}{*}{0.0014} & \multirow{2}{*}{$<0.0001$} \\
\hline & False & $592(44.4 \%)$ & $511(38.3 \%)$ & & \\
\hline \multirow{3}{*}{$\begin{array}{l}\text { If I am having a dessert at home, I try } \\
\text { to have something low in fat }\end{array}$} & True & $433(32.5 \%)$ & $483(36.2 \%)$ & \multirow{3}{*}{0.1249} & \multirow{3}{*}{$<0.0001$} \\
\hline & False & $679(50.9 \%)$ & $642(48.2 \%)$ & & \\
\hline & Not applicable & $221(16.6 \%)$ & $208(15.6 \%)$ & & \\
\hline \multirow{2}{*}{$\begin{array}{l}\text { I usually eat at least one serving of } \\
\text { vegetables (excluding potatoes) or } \\
\text { salad with my evening meal }\end{array}$} & True & $1006(75.5 \%)$ & $1064(79.8 \%)$ & \multirow{2}{*}{0.0070} & \multirow{2}{*}{$<0.0001$} \\
\hline & False & $327(24.5 \%)$ & $269(20.2 \%)$ & & \\
\hline \multirow{3}{*}{$\begin{array}{l}\text { When I put butter or margarine on } \\
\text { bread, I usually spread it thinly }\end{array}$} & True & $916(68.7 \%)$ & $912(68.4 \%)$ & \multirow{3}{*}{0.7811} & \multirow{3}{*}{0.7604} \\
\hline & False & $198(14.9 \%)$ & $190(14.3 \%)$ & & \\
\hline & Not applicable & $219(16.4 \%)$ & $231(17.3 \%)$ & & \\
\hline \multirow{3}{*}{$\begin{array}{l}\text { If I have a packed lunch, I usually } \\
\text { include some chocolate and/or biscuits }\end{array}$} & True & $435(32.6 \%)$ & $308(23.1 \%)$ & \multirow{3}{*}{$<0.0001$} & \multirow{3}{*}{$<0.0001$} \\
\hline & False & $751(56.3 \%)$ & $795(59.6 \%)$ & & \\
\hline & Not applicable & $147(11.1 \%)$ & $230(17.3 \%)$ & & \\
\hline \multirow{3}{*}{ I often have cream on desserts } & True & $288(21.6 \%)$ & $291(21.8 \%)$ & \multirow{3}{*}{0.7115} & \multirow{3}{*}{$<0.0001$} \\
\hline & False & $886(66.5 \%)$ & $870(65.3 \%)$ & & \\
\hline & Not applicable & $159(11.9 \%)$ & $172(12.9 \%)$ & & \\
\hline
\end{tabular}

*AFHC-Adolescents' Food Habits Checklist [37]; ** chi-square test (for the comparison of the frequency of specific answers); ${ }^{* * *}$ McNemar's test (for longitudinal analysis).

The food consumption habits assessed while using AFHC in the period before the COVID-19 pandemic and during the COVID-19 pandemic, as declared by the adolescents from the DAY-19 Study cohort, are presented in Table 3. During the COVID-19 pandemic, the number of respondents who declared making sure that they eat at least one serving of fruit a day increased $(82.3 \%$ vs. $86.3 \%, p<0.004)$ and eating at least three servings of fruit most days increased $(47.4 \%$ vs. $56.6 \%, p<0.0001)$, while the number of respondents who declared making sure that they eat at least one serving of vegetables or salad a day also increased $(71.2 \%$ vs. $78.4 \%, p<0.0001)$. Moreover, the number of respondents who declared trying to ensure that they eat plenty of fruit and vegetables increased $(75.5 \%$ vs. $82.2 \%$, $p<0.0001$ ), while the number of respondents who declared often choosing a fruit when they have a snack between meals increased $(45.9 \%$ vs. $51.4 \%, p<0.05)$ and the number of respondents who declared eating at least three servings of fruit most days also increased $(47.4 \%$ vs. $56.6 \%, p<0.0001)$. At the same time, the number of respondents who declared that they generally try to have a healthy diet also increased $(72.2 \%$ vs. $80.1 \%, p<0.0001)$. The longitudinal analysis confirmed significant changes of food consumption habits in the period of the COVID-19 pandemic, which was stated for all the habits except for eating sweet snacks between meals. 
Table 3. The food consumption habits assessed while using Adolescents' Food Habits Checklist (AFHC) in the period before the COVID-19 pandemic and during the COVID-19 pandemic, as declared by the adolescents from the Diet and Activity of Youth during COVID-19 (DAY-19) Study cohort $(n=1333)$.

\begin{tabular}{|c|c|c|c|c|c|}
\hline \multicolumn{2}{|c|}{ Food Consumption Habits Assessed within AFHC * } & \multirow{3}{*}{$\begin{array}{c}\begin{array}{c}\text { Before COVID-19 } \\
\text { Pandemic }\end{array} \\
953(71.5 \%) \\
380(28.5 \%) \\
\end{array}$} & \multirow{3}{*}{$\begin{array}{c}\begin{array}{c}\text { During COVID-19 } \\
\text { Pandemic }\end{array} \\
963(72.2 \%) \\
370(27.8 \%) \\
\end{array}$} & \multirow{3}{*}{$\begin{array}{c}p^{* *} \\
0.6663\end{array}$} & \multirow{3}{*}{$\begin{array}{c}p^{* * *} \\
<0.0001\end{array}$} \\
\hline I usually eat a dessert or pudding if & True & & & & \\
\hline there is one available & False & & & & \\
\hline \multirow{2}{*}{$\begin{array}{l}\text { I make sure I eat at least one serving of } \\
\text { fruit a day }\end{array}$} & True & $1097(82.3 \%)$ & $1151(86.3 \%)$ & \multirow{2}{*}{0.0040} & \multirow{2}{*}{$<0.0001$} \\
\hline & False & $236(17.7 \%)$ & $182(13.7 \%)$ & & \\
\hline \multirow{3}{*}{$\begin{array}{l}\text { I avoid eating lots of sausages } \\
\text { and burgers }\end{array}$} & True & $783(58.8 \%)$ & $809(60.7 \%)$ & \multirow{3}{*}{0.4219} & \multirow{3}{*}{$<0.0001$} \\
\hline & False & $343(25.7 \%)$ & $314(23.5 \%)$ & & \\
\hline & Not applicable & $207(15.5 \%)$ & $210(15.8 \%)$ & & \\
\hline \multirow{2}{*}{$\begin{array}{l}\text { I make sure I eat at least one serving of } \\
\text { vegetables or salad a day }\end{array}$} & True & $949(71.2 \%)$ & $1045(78.4 \%)$ & \multirow{2}{*}{$<0.0001$} & \multirow{2}{*}{$<0.0001$} \\
\hline & False & $384(28.8 \%)$ & $288(21.6 \%)$ & & \\
\hline \multirow{2}{*}{$\begin{array}{l}\text { I try to ensure I eat plenty of fruit } \\
\text { and vegetables }\end{array}$} & True & $1009(75.7 \%)$ & $1096(82.2 \%)$ & \multirow{2}{*}{$<0.0001$} & \multirow{2}{*}{$<0.0001$} \\
\hline & False & $324(24.3 \%)$ & $237(17.8 \%)$ & & \\
\hline \multirow{2}{*}{ I often eat sweet snacks between meals } & True & $623(46.7 \%)$ & $641(48.1 \%)$ & \multirow{2}{*}{0.4853} & \multirow{2}{*}{0.2954} \\
\hline & False & $710(53.3 \%)$ & $692(51.9 \%)$ & & \\
\hline \multirow{3}{*}{$\begin{array}{l}\text { When I have a snack between meals, I } \\
\text { often choose fruit }\end{array}$} & True & $611(45.9 \%)$ & $686(51.4 \%)$ & \multirow{3}{*}{0.0111} & \multirow{3}{*}{$<0.0001$} \\
\hline & False & $558(41.8 \%)$ & $489(36.7 \%)$ & & \\
\hline & Not applicable & $164(12.3 \%)$ & $158(11.9 \%)$ & & \\
\hline \multirow{2}{*}{$\begin{array}{l}\text { I eat at least three servings of fruit } \\
\text { most days }\end{array}$} & True & $632(47.4 \%)$ & $754(56.6 \%)$ & \multirow{2}{*}{$<0.0001$} & \multirow{2}{*}{$<0.0001$} \\
\hline & False & $701(52.6 \%)$ & $579(43.4 \%)$ & & \\
\hline \multirow{2}{*}{ I generally try to have a healthy diet } & True & $963(72.2 \%)$ & $1068(80.1 \%)$ & \multirow{2}{*}{$<0.0001$} & \multirow{2}{*}{$<0.0001$} \\
\hline & False & $370(27.8 \%)$ & $265(19.9 \%)$ & & \\
\hline
\end{tabular}

* AFHC-Adolescents' Food Habits Checklist [37]; ${ }^{* *}$ chi-square test (for the comparison of the frequency of specific answers); ${ }^{* * *}$ McNemar's test (for longitudinal analysis).

\subsection{Comparison of Results Associated with Body Mass Changes}

The food purchase habits assessed while using AFHC in the period during the COVID19 pandemic, as declared by the adolescents from the DAY-19 Study cohort in sub-groups of adolescents declaring decreased, stable, and increased body mass during the COVID-19 pandemic are presented in Table 4. A lower number of respondents who reported increased body mass, when compared with those who reported decreased body mass, declared often choosing a low-fat option while having lunch away from home (28.3\% vs. $46.1 \%$, $p<0.0001)$, rarely eating takeaway meals $(80.1 \%$ vs. $86.8 \%, p<0.05)$, choosing a low-fat crisps brand ( $23.9 \%$ vs. $28.8 \%, p<0.001)$, and choosing a diet soft drink ( $34.9 \%$ vs. $58.0 \%$, $p<0.0001)$, as well as usually choosing the healthiest dessert or pudding in a restaurant $(15.8 \%$ vs. $25.5 \%, p<0001)$. At the same time, a higher number of respondents who reported increased body mass, when compared with those who reported decreased body mass, declared often buying pastries or cakes $(28.3 \%$ vs. $20.2 \%, p<0.05)$.

Table 4. The food purchase habits assessed while using Adolescents' Food Habits Checklist (AFHC) in the period during the COVID-19 pandemic, as declared by the adolescents from the Diet and Activity of Youth during COVID-19 (DAY-19) Study cohort $(n=1333)$ in sub-groups of adolescents declaring decreased, stable, and increased body mass during the COVID-19 pandemic.

\begin{tabular}{|c|c|c|c|c|c|}
\hline \multicolumn{2}{|c|}{ Food Purchase Habits Assessed within AFHC * } & $\begin{array}{l}\text { Decreased Body Mass } \\
\text { during COVID-19 } \\
\text { Pandemic } \\
(n=243)\end{array}$ & $\begin{array}{l}\text { Stable Body Mass during } \\
\text { COVID-19 Pandemic } \\
(n=552)\end{array}$ & $\begin{array}{l}\text { Increased Body Mass } \\
\text { during COVID-19 } \\
\text { Pandemic } \\
(n=538)\end{array}$ & $p^{* *}$ \\
\hline $\begin{array}{l}\text { If I am having lunch away from home, } \\
\text { I often choose a low-fat option }\end{array}$ & $\begin{array}{c}\text { True } \\
\text { False } \\
\text { Not applicable }\end{array}$ & $\begin{array}{l}112(46.1 \%) \\
49(20.2 \%) \\
82(33.7 \%)\end{array}$ & $\begin{array}{l}189(34.3 \%) \\
149(26.9 \%) \\
214(38.8 \%)\end{array}$ & $\begin{array}{l}152(28.3 \%) \\
167(31.0 \%) \\
219(40.7 \%)\end{array}$ & $<0.0001$ \\
\hline $\begin{array}{l}\text { If I am buying crisps, I often choose a } \\
\text { low-fat brand }\end{array}$ & $\begin{array}{c}\text { True } \\
\text { False } \\
\text { Not applicable }\end{array}$ & $\begin{array}{l}70(28.8 \%) \\
106(43.6 \%) \\
67(27.6 \%)\end{array}$ & $\begin{array}{l}132(23.9 \%) \\
299(54.2 \%) \\
121(21.9 \%)\end{array}$ & $\begin{array}{l}129(23.9 \%) \\
321(59.7 \%) \\
88(16.4 \%)\end{array}$ & 0.0004 \\
\hline
\end{tabular}


Table 4. Cont.

\begin{tabular}{|c|c|c|c|c|c|}
\hline \multicolumn{2}{|c|}{ Food Purchase Habits Assessed within AFHC * } & $\begin{array}{c}\text { Decreased Body Mass } \\
\text { during COVID-19 } \\
\text { Pandemic } \\
(n=243)\end{array}$ & $\begin{array}{l}\text { Stable Body Mass during } \\
\text { COVID-19 Pandemic } \\
(n=552)\end{array}$ & $\begin{array}{c}\text { Increased Body Mass } \\
\text { during COVID-19 } \\
\text { Pandemic } \\
(n=538)\end{array}$ & $p^{* *}$ \\
\hline I often buy pastries or cakes & $\begin{array}{l}\text { True } \\
\text { False }\end{array}$ & $\begin{array}{c}49(20.2 \%) \\
194(79.8 \%) \\
\end{array}$ & $\begin{array}{l}121(21.9 \%) \\
431(78.1 \%) \\
\end{array}$ & $\begin{array}{l}152(28.3 \%) \\
386(71.7 \%) \\
\end{array}$ & 0.0139 \\
\hline I rarely eat takeaway meals & $\begin{array}{l}\text { True } \\
\text { False }\end{array}$ & $\begin{array}{l}211(86.8 \%) \\
32(13.2 \%)\end{array}$ & $\begin{array}{l}466(84.4 \%) \\
86(15.6 \%)\end{array}$ & $\begin{array}{l}431(80.1 \%) \\
107(19.9 \%)\end{array}$ & 0.0384 \\
\hline $\begin{array}{l}\text { When I am buying a soft drink, I } \\
\text { usually choose a diet drink }\end{array}$ & $\begin{array}{c}\text { True } \\
\text { False }\end{array}$ & $\begin{array}{l}141(58.0 \%) \\
102(42.0 \%) \\
\end{array}$ & $\begin{array}{l}201(36.4 \%) \\
351(63.6 \%) \\
\end{array}$ & $\begin{array}{l}188(34.9 \%) \\
350(65.1 \%) \\
\end{array}$ & $<0.0001$ \\
\hline $\begin{array}{l}\text { If I am having a dessert or pudding in } \\
\text { a restaurant, I usually choose the } \\
\text { healthiest one }\end{array}$ & $\begin{array}{c}\text { True } \\
\text { False } \\
\text { Not applicable } \\
\end{array}$ & $\begin{array}{c}62(25.5 \%) \\
61(25.1 \%) \\
120(49.4 \%) \\
\end{array}$ & $\begin{array}{c}79(14.3 \%) \\
235(42.6 \%) \\
238(43.1 \%) \\
\end{array}$ & $\begin{array}{c}85(15.8 \%) \\
239(44.4 \%) \\
214(39.8 \%) \\
\end{array}$ & $<0.0001$ \\
\hline
\end{tabular}

* AFHC-Adolescents' Food Habits Checklist [37]; ** chi-square test.

The food preparation habits assessed while using AFHC in the period during the COVID-19 pandemic, as declared by the adolescents from the DAY-19 Study cohort, in sub-groups of adolescents declaring decreased, stable, and increased body mass during the COVID-19 pandemic are presented in Table 5. A lower number of respondents who reported increased body mass, when compared with those who reported decreased body mass, declared avoiding eating fried foods $(34.9 \%$ vs. $54.7 \%, p<0.0001)$, trying to keep their overall fat intake down $(53.7 \%$ vs. $72.8 \%, p<0.0001)$, trying to keep their overall sugar intake down $(57.6 \%$ vs. $73.7 \%, p<0.0001)$, trying to have something low in fat while having a dessert at home ( $32.5 \%$ vs. $43.2 \%, p<0.0001)$, usually eating at least one serving of vegetables or salad with evening meal $(76.6 \%$ vs. $83.5 \%, p<0.05)$, and usually including some chocolate and/or biscuits while having a packed lunch $(26.7 \%$ vs. $46.1 \%, p<0.0001)$.

Table 5. The food preparation habits assessed while using Adolescents' Food Habits Checklist (AFHC) in the period during the COVID-19 pandemic, as declared by the adolescents from the Diet and Activity of Youth during COVID-19 (DAY-19) Study cohort $(n=1333)$ in sub-groups of adolescents declaring decreased, stable, and increased body mass during the COVID-19 pandemic.

\begin{tabular}{|c|c|c|c|c|c|}
\hline \multicolumn{2}{|c|}{ Food Preparation Habits Assessed within AFHC * } & \multirow{2}{*}{$\begin{array}{c}\begin{array}{c}\text { Decreased Body Mass } \\
\text { during COVID-19 } \\
\text { Pandemic } \\
(\boldsymbol{n}=243)\end{array} \\
133(54.7 \%) \\
110(45.3 \%)\end{array}$} & \multirow{2}{*}{$\begin{array}{c}\begin{array}{c}\text { Stable Body Mass during } \\
\text { COVID-19 Pandemic } \\
(\boldsymbol{n}=\mathbf{5 5 2})\end{array} \\
215(38.9 \%) \\
337(61.1 \%)\end{array}$} & \multirow{2}{*}{$\begin{array}{c}\begin{array}{c}\text { Increased Body Mass } \\
\text { during COVID-19 } \\
\text { Pandemic } \\
(\boldsymbol{n}=\mathbf{5 3 8})\end{array} \\
188(34.9 \%) \\
350(65.1 \%)\end{array}$} & \multirow{2}{*}{$\begin{array}{c}p^{* *} \\
<0.0001\end{array}$} \\
\hline I usually avoid eating fried foods & $\begin{array}{l}\text { True } \\
\text { False }\end{array}$ & & & & \\
\hline $\begin{array}{l}\text { I try to keep my overall fat } \\
\text { intake down }\end{array}$ & $\begin{array}{l}\text { True } \\
\text { False }\end{array}$ & $\begin{array}{l}177(72.8 \%) \\
66(27.2 \%)\end{array}$ & $\begin{array}{l}334(60.5 \%) \\
218(39.5 \%)\end{array}$ & $\begin{array}{l}289(53.7 \%) \\
249(46.3 \%)\end{array}$ & $<0.0001$ \\
\hline $\begin{array}{l}\text { I try to keep my overall sugar } \\
\text { intake down }\end{array}$ & $\begin{array}{l}\text { True } \\
\text { False }\end{array}$ & $\begin{array}{l}179(73.7 \%) \\
64(26.3 \%)\end{array}$ & $\begin{array}{l}333(60.3 \%) \\
219(39.7 \%)\end{array}$ & $\begin{array}{l}310(57.6 \%) \\
228(42.4 \%)\end{array}$ & $<0.0001$ \\
\hline $\begin{array}{l}\text { If I am having a dessert at home, I try } \\
\text { to have something low in fat }\end{array}$ & $\begin{array}{c}\text { True } \\
\text { False } \\
\text { Not applicable }\end{array}$ & $\begin{array}{l}105(43.2 \%) \\
82(33.8 \%) \\
56(23.0 \%)\end{array}$ & $\begin{array}{l}203(36.8 \%) \\
270(48.9 \%) \\
79(14.3 \%)\end{array}$ & $\begin{array}{c}175(32.5 \%) \\
290(53.9 \%) \\
73(13.6 \%)\end{array}$ & $<0.0001$ \\
\hline $\begin{array}{l}\text { I usually eat at least one serving of } \\
\text { vegetables (excluding potatoes) or } \\
\text { salad with my evening meal }\end{array}$ & $\begin{array}{l}\text { True } \\
\text { False }\end{array}$ & $\begin{array}{c}203(83.5 \%) \\
40(16.5 \%)\end{array}$ & $\begin{array}{l}449(81.3 \%) \\
103(18.7 \%)\end{array}$ & $\begin{array}{l}412(76.6 \%) \\
126(23.4 \%)\end{array}$ & 0.0411 \\
\hline $\begin{array}{l}\text { When I put butter or margarine on } \\
\text { bread, I usually spread it thinly }\end{array}$ & $\begin{array}{c}\text { True } \\
\text { False } \\
\text { Not applicable }\end{array}$ & $\begin{array}{l}158(65.0 \%) \\
35(14.4 \%) \\
50(20.6 \%)\end{array}$ & $\begin{array}{l}379(68.6 \%) \\
74(13.5 \%) \\
99(17.9 \%)\end{array}$ & $\begin{array}{l}375(69.7 \%) \\
81(15.1 \%) \\
82(15.2 \%)\end{array}$ & 0.4036 \\
\hline $\begin{array}{l}\text { If I have a packed lunch, I usually } \\
\text { include some chocolate } \\
\text { and/or biscuits }\end{array}$ & $\begin{array}{c}\text { True } \\
\text { False } \\
\text { Not applicable }\end{array}$ & $\begin{array}{l}112(46.1 \%) \\
49(20.2 \%) \\
82(33.7 \%)\end{array}$ & $\begin{array}{l}121(21.9 \%) \\
339(61.4 \%) \\
92(16.7 \%)\end{array}$ & $\begin{array}{l}144(26.7 \%) \\
299(55.6 \%) \\
95(17.7 \%)\end{array}$ & $<0.0001$ \\
\hline I often have cream on desserts & $\begin{array}{c}\text { True } \\
\text { False } \\
\text { Not applicable }\end{array}$ & $\begin{array}{l}49(20.2 \%) \\
157(64.6 \%) \\
37(15.2 \%)\end{array}$ & $\begin{array}{l}110(19.9 \%) \\
374(67.8 \%) \\
68(12.3 \%)\end{array}$ & $\begin{array}{c}132(24.5 \%) \\
339(63.0 \%) \\
67(12.5 \%)\end{array}$ & 0.2642 \\
\hline
\end{tabular}

* AFHC-Adolescents' Food Habits Checklist [37]; ** chi-square test.

The food consumption habits assessed while using AFHC in the period during the COVID-19 pandemic, as declared by the adolescents from the DAY-19 Study cohort, in sub-groups of adolescents declaring decreased, stable, and increased body mass during the COVID-19 pandemic are presented in Table 6. A higher number of respondents who reported increased body mass, when compared with those who reported decreased body mass, declared usually eating a dessert or pudding if there is one available $(73.8 \%$ vs. $64.2 \%$, 
$p<0.01)$ and often eating sweet snacks between meals (55.6\% vs. $32.1 \%, p<0.0001)$. At the same time, lower number of respondents who reported increased body mass, when compared with those who reported decreased body mass, declared avoiding eating lots of sausages and burgers (57.8\% vs. $65.0 \%, p<0.001)$, making sure they eat at least one serving of vegetables or salad a day $(74.9 \%$ vs. $85.6 \%, p<0.01)$, trying to ensure they eat plenty of fruit and vegetables $(78.6 \%$ vs. $86.0 \%, p<0.05)$, often choosing fruit while having a snack between meals $(48.9 \%$ vs. $56.4 \%, p<0.0001)$, eating at least three servings of fruit most days $(53.3 \%$ vs. $69.1 \%, p<0.0001)$, and generally trying to have a healthy diet.

Table 6. The food consumption habits assessed while using Adolescents' Food Habits Checklist (AFHC) in the period during the COVID-19 pandemic, as declared by the adolescents from the Diet and Activity of Youth during COVID-19 (DAY-19) Study cohort $(n=1333)$ in sub-groups of adolescents declaring decreased, stable, and increased body mass during the COVID-19 pandemic.

\begin{tabular}{|c|c|c|c|c|c|}
\hline \multicolumn{2}{|c|}{ Food Consumption Habits Assessed within AFHC * } & \multirow{2}{*}{$\begin{array}{c}\begin{array}{c}\text { Decreased Body Mass } \\
\text { during COVID-19 } \\
\text { Pandemic } \\
(\boldsymbol{n}=\mathbf{2 4 3})\end{array} \\
156(64.2 \%) \\
87(35.8 \%) \\
\end{array}$} & \multirow{2}{*}{$\begin{array}{c}\begin{array}{c}\text { Stable Body Mass during } \\
\text { COVID-19 Pandemic } \\
(n=552)\end{array} \\
410(74.3 \%) \\
142(25.7 \%) \\
\end{array}$} & \multirow{2}{*}{$\begin{array}{c}\begin{array}{c}\text { Increased Body Mass } \\
\text { during COVID-19 } \\
\text { Pandemic } \\
(\boldsymbol{n}=\mathbf{5 3 8})\end{array} \\
397(73.8 \%) \\
141(26.2 \%) \\
\end{array}$} & \multirow{2}{*}{$\begin{array}{c}p^{* *} \\
0.0081\end{array}$} \\
\hline $\begin{array}{l}\text { I usually eat a dessert or pudding if } \\
\text { there is one available }\end{array}$ & $\begin{array}{l}\text { True } \\
\text { False }\end{array}$ & & & & \\
\hline $\begin{array}{l}\text { I make sure I eat at least one serving } \\
\text { of fruit a day }\end{array}$ & $\begin{array}{l}\text { True } \\
\text { False }\end{array}$ & $\begin{array}{l}212(87.3 \%) \\
31(12.7 \%)\end{array}$ & $\begin{array}{l}482(87.3 \%) \\
70(12.7 \%)\end{array}$ & $\begin{array}{l}457(84.9 \%) \\
81(15.1 \%)\end{array}$ & 0.4709 \\
\hline $\begin{array}{l}\text { I avoid eating lots of sausages } \\
\text { and burgers }\end{array}$ & $\begin{array}{c}\text { True } \\
\text { False } \\
\text { Not applicable }\end{array}$ & $\begin{array}{l}158(65.0 \%) \\
32(13.2 \%) \\
53(21.8 \%)\end{array}$ & $\begin{array}{l}340(61.6 \%) \\
132(23.9 \%) \\
80(14.5 \%)\end{array}$ & $\begin{array}{l}311(57.8 \%) \\
150(27.9 \%) \\
77(14.3 \%)\end{array}$ & $<0.0001$ \\
\hline $\begin{array}{c}\text { I make sure I eat at least one serving } \\
\text { of vegetables or salad a day }\end{array}$ & $\begin{array}{l}\text { True } \\
\text { False }\end{array}$ & $\begin{array}{l}208(85.6 \%) \\
35(14.4 \%)\end{array}$ & $\begin{array}{l}434(78.6 \%) \\
118(21.4 \%)\end{array}$ & $\begin{array}{l}403(74.9 \%) \\
135(25.1 \%)\end{array}$ & 0.0035 \\
\hline $\begin{array}{l}\text { I try to ensure I eat plenty of fruit } \\
\text { and vegetables }\end{array}$ & $\begin{array}{l}\text { True } \\
\text { False }\end{array}$ & $\begin{array}{l}209(86.0 \%) \\
34(14.0 \%)\end{array}$ & $\begin{array}{l}464(84.1 \%) \\
88(15.9 \%)\end{array}$ & $\begin{array}{l}423(78.6 \%) \\
115(21.4 \%)\end{array}$ & 0.0148 \\
\hline $\begin{array}{l}\text { I often eat sweet snacks } \\
\text { between meals }\end{array}$ & $\begin{array}{l}\text { True } \\
\text { False }\end{array}$ & $\begin{array}{c}78(32.1 \%) \\
165(67.9 \%)\end{array}$ & $\begin{array}{l}264(47.8 \%) \\
288(52.2 \%)\end{array}$ & $\begin{array}{l}299(55.6 \%) \\
239(44.4 \%) \\
\end{array}$ & $<0.0001$ \\
\hline $\begin{array}{l}\text { When I have a snack between meals, I } \\
\text { often choose fruit }\end{array}$ & $\begin{array}{c}\text { True } \\
\text { False } \\
\text { Not applicable }\end{array}$ & $\begin{array}{l}137(56.4 \%) \\
60(24.7 \%) \\
46(18.9 \%)\end{array}$ & $\begin{array}{l}286(51.8 \%) \\
207(37.5 \%) \\
59(10.7 \%)\end{array}$ & $\begin{array}{c}263(48.9 \%) \\
222(41.3 \%) \\
53(9.8 \%)\end{array}$ & $<0.0001$ \\
\hline $\begin{array}{l}\text { I eat at least three servings of fruit } \\
\text { most days }\end{array}$ & $\begin{array}{l}\text { True } \\
\text { False }\end{array}$ & $\begin{array}{l}168(69.1 \%) \\
75(30.9 \%)\end{array}$ & $\begin{array}{l}299(54.2 \%) \\
253(45.8 \%)\end{array}$ & $\begin{array}{l}287(53.3 \%) \\
251(46.7 \%)\end{array}$ & $<0.0001$ \\
\hline I generally try to have a healthy diet & $\begin{array}{l}\text { True } \\
\text { False }\end{array}$ & $\begin{array}{l}219(90.1 \%) \\
24(9.9 \%)\end{array}$ & $\begin{array}{l}446(80.8 \%) \\
106(19.2 \%)\end{array}$ & $\begin{array}{l}403(74.9 \%) \\
135(25.1 \%)\end{array}$ & $<0.0001$ \\
\hline
\end{tabular}

* AFHC-Adolescents' Food Habits Checklist [37]; ** chi-square test.

\subsection{Comparison of Results Associated with Physcial Activity Changes}

The food purchase habits assessed while using AFHC in the period during the COVID19 pandemic, as declared by the adolescents from the DAY-19 Study cohort, in sub-groups of adolescents declaring decreased, stable, and increased physical activity during the COVID-19 pandemic are presented in Table 7. A lower number of respondents who reported decreased physical activity, when compared with those who reported increased physical activity, declared often choosing a low-fat option while having lunch away from home $(27.9 \%$ vs. $38.8 \%, p<0.01)$, often choosing a low-fat crisps brand $(20.8 \%$ vs. $25.5 \%$, $p<0.001)$, usually choosing a diet soft drink $(28.2 \%$ vs. $48.2 \%, p<0.0001)$, and usually choosing the healthiest dessert or pudding in a restaurant $(29.7 \%$ vs. $37.2 \%, p<0.0001)$. At the same time, a higher number of respondents who reported decreased physical activity, when compared with those who reported increased physical activity, declared often buying pastries or cakes $(27.1 \%$ vs. $25.2 \%, p<0.05)$. 
Table 7. The food purchase habits assessed while using Adolescents' Food Habits Checklist (AFHC) in the period during the COVID-19 pandemic, as declared by the adolescents from the Diet and Activity of Youth during COVID-19 (DAY-19) Study cohort $(n=1333)$ in sub-groups of adolescents declaring decreased, stable, and increased physical activity during the COVID-19 pandemic.

\begin{tabular}{|c|c|c|c|c|c|}
\hline \multicolumn{2}{|c|}{ Food Purchase Habits Assessed within AFHC * } & \multirow{2}{*}{$\begin{array}{c}\begin{array}{c}\text { Decreased Physical } \\
\text { Activity during } \\
\text { COVID-19 Pandemic } \\
(\boldsymbol{n}=\mathbf{4 7 6})\end{array} \\
133(27.9 \%) \\
156(32.8 \%) \\
187(39.4 \%) \\
\end{array}$} & \multirow{2}{*}{$\begin{array}{c}\begin{array}{c}\text { Stable Physical Activity } \\
\text { during COVID-19 } \\
\text { Pandemic } \\
(\boldsymbol{n}=390)\end{array} \\
139(35.6 \%) \\
96(24.6 \%) \\
155(39.8 \%)\end{array}$} & \multirow{2}{*}{$\begin{array}{c}\begin{array}{c}\text { Increased Physical } \\
\text { Activity during } \\
\text { COVID-19 Pandemic } \\
(\boldsymbol{n}=467)\end{array} \\
181(38.8 \%) \\
113(24.2 \%) \\
173(37.0 \%) \\
\end{array}$} & \multirow{2}{*}{$\begin{array}{c}p^{* *} \\
0.0020\end{array}$} \\
\hline $\begin{array}{l}\text { If I am having lunch away from home, } \\
\text { I often choose a low-fat option }\end{array}$ & $\begin{array}{c}\text { True } \\
\text { False } \\
\text { Not applicable }\end{array}$ & & & & \\
\hline $\begin{array}{l}\text { If I am buying crisps, I often choose a } \\
\text { low-fat brand }\end{array}$ & $\begin{array}{c}\text { True } \\
\text { False } \\
\text { Not applicable }\end{array}$ & $\begin{array}{l}99(20.8 \%) \\
309(64.9 \%) \\
68(14.3 \%)\end{array}$ & $\begin{array}{l}113(28.9 \%) \\
193(49.6 \%) \\
84(21.5 \%)\end{array}$ & $\begin{array}{l}119(25.5 \%) \\
224(47.9 \%) \\
124(26.6 \%)\end{array}$ & $<0.0001$ \\
\hline I often buy pastries or cakes & $\begin{array}{l}\text { True } \\
\text { False }\end{array}$ & $\begin{array}{l}129(27.1 \%) \\
347(72.9 \%)\end{array}$ & $\begin{array}{c}75(19.2 \%) \\
315(80.8 \%)\end{array}$ & $\begin{array}{l}118(25.3 \%) \\
349(74.7 \%)\end{array}$ & 0.0209 \\
\hline I rarely eat takeaway meals & $\begin{array}{l}\text { True } \\
\text { False }\end{array}$ & $\begin{array}{c}398(83.6 \%) \\
78(16.4 \%)\end{array}$ & $\begin{array}{c}333(85.4 \%) \\
57(14.6 \%)\end{array}$ & $\begin{array}{c}377(80.7 \%) \\
90(19.3 \%)\end{array}$ & 0.1815 \\
\hline $\begin{array}{c}\text { When I am buying a soft drink, I } \\
\text { usually choose a diet drink }\end{array}$ & $\begin{array}{l}\text { True } \\
\text { False }\end{array}$ & $\begin{array}{l}134(28.2 \%) \\
342(71.8 \%)\end{array}$ & $\begin{array}{l}171(43.8 \%) \\
219(56.2 \%)\end{array}$ & $\begin{array}{l}225(48.2 \%) \\
242(51.8 \%)\end{array}$ & $<0.0001$ \\
\hline $\begin{array}{l}\text { If I am having a dessert or pudding in } \\
\text { a restaurant, I usually choose the } \\
\text { healthiest one }\end{array}$ & $\begin{array}{c}\text { True } \\
\text { False } \\
\text { Not applicable }\end{array}$ & $\begin{array}{l}141(29.7 \%) \\
278(58.4 \%) \\
57(11.9 \%)\end{array}$ & $\begin{array}{l}168(43.1 \%) \\
161(41.3 \%) \\
61(15.6 \%)\end{array}$ & $\begin{array}{l}174(37.2 \%) \\
203(43.5 \%) \\
90(19.3 \%)\end{array}$ & $<0.0001$ \\
\hline
\end{tabular}

* AFHC-Adolescents' Food Habits Checklist [37]; ** chi-square test.

The food preparation habits assessed while using AFHC in the period during the COVID-19 pandemic, as declared by the adolescents from the DAY-19 Study cohort, in subgroups of adolescents declaring decreased, stable, and increased physical activity during the COVID-19 pandemic are presented in Table 8. A lower number of respondents who reported decreased physical activity, when compared with those who reported increased physical activity, declared usually avoiding eating fried foods $(32.4 \%$ vs. $46.3 \%, p<0.0001)$, trying to keep their overall fat intake down $(48.9 \%$ vs. $65.7 \%, p<0.0001)$, trying to keep their overall sugar intake down $(50.8 \%$ vs. $68.5 \%, p<0.0001)$, trying to have something low in fat while having a dessert at home $(29.6 \%$ vs. $37.2 \%, p<0.0001)$, and usually eating at least one serving of vegetables or salad with evening meal $(75.8 \%$ vs. $84.6 \%, p<0.01)$. At the same time, a higher number of respondents who reported decreased physical activity, when compared with those who reported increased physical activity, declared usually spreading butter or margarine on bread thinly ( $69.3 \%$ vs. $65.1 \%, p<0.05)$, and usually including some chocolate and/or biscuits to a packed lunch $(27.3 \%$ vs. $18.6 \%, p<0.05)$.

Table 8. The food preparation habits assessed while using Adolescents' Food Habits Checklist (AFHC) in the period during the COVID-19 pandemic, as declared by the adolescents from the Diet and Activity of Youth during COVID-19 (DAY-19) Study cohort $(n=1333)$ in sub-groups of adolescents declaring decreased, stable, and increased physical activity during the COVID-19 pandemic.

\begin{tabular}{|c|c|c|c|c|c|}
\hline \multicolumn{2}{|c|}{ Food Preparation Habits Assessed within AFHC * } & $\begin{array}{l}\text { Decreased Physical } \\
\text { Activity during } \\
\text { COVID-19 Pandemic } \\
(n=476)\end{array}$ & $\begin{array}{l}\text { Stable Physical Activity } \\
\text { during COVID-19 } \\
\text { Pandemic } \\
(n=390)\end{array}$ & $\begin{array}{c}\text { Increased Physical } \\
\text { Activity during } \\
\text { COVID-19 Pandemic } \\
(n=467)\end{array}$ & $p^{* *}$ \\
\hline I usually avoid eating fried foods & $\begin{array}{l}\text { True } \\
\text { False }\end{array}$ & $\begin{array}{l}154(32.4 \%) \\
322(67.6 \%)\end{array}$ & $\begin{array}{l}166(42.6 \%) \\
224(57.4 \%)\end{array}$ & $\begin{array}{l}216(46.3 \%) \\
251(53.7 \%)\end{array}$ & $<0.0001$ \\
\hline $\begin{array}{l}\text { I try to keep my overall fat } \\
\text { intake down }\end{array}$ & $\begin{array}{l}\text { True } \\
\text { False }\end{array}$ & $\begin{array}{l}233(48.9 \%) \\
243(51.1 \%)\end{array}$ & $\begin{array}{l}260(66.7 \%) \\
130(33.3 \%)\end{array}$ & $\begin{array}{l}307(65.7 \%) \\
160(34.2 \%)\end{array}$ & $<0.0001$ \\
\hline $\begin{array}{c}\text { I try to keep my overall sugar } \\
\text { intake down }\end{array}$ & $\begin{array}{l}\text { True } \\
\text { False }\end{array}$ & $\begin{array}{l}242(50.8 \%) \\
234(49.2 \%)\end{array}$ & $\begin{array}{l}260(66.7 \%) \\
130(33.3 \%)\end{array}$ & $\begin{array}{l}320(68.5 \%) \\
147(31.5 \%)\end{array}$ & $<0.0001$ \\
\hline $\begin{array}{l}\text { If I am having a dessert at home, I try } \\
\text { to have something low in fat }\end{array}$ & $\begin{array}{c}\text { True } \\
\text { False } \\
\text { Not applicable }\end{array}$ & $\begin{array}{l}141(29.6 \%) \\
278(58.4 \%) \\
57(12.0 \%)\end{array}$ & $\begin{array}{l}168(43.1 \%) \\
161(41.3 \%) \\
61(15.6 \%)\end{array}$ & $\begin{array}{l}174(37.2 \%) \\
203(43.5 \%) \\
90(19.3 \%)\end{array}$ & $<0.0001$ \\
\hline $\begin{array}{l}\text { I usually eat at least one serving of } \\
\text { vegetables (excluding potatoes) or } \\
\text { salad with my evening meal }\end{array}$ & $\begin{array}{l}\text { True } \\
\text { False }\end{array}$ & $\begin{array}{l}361(75.8 \%) \\
115(24.2 \%)\end{array}$ & $\begin{array}{c}308(79.0 \%) \\
82(21.0 \%)\end{array}$ & $\begin{array}{l}395(84.6 \%) \\
72(15.4 \%)\end{array}$ & 0.0033 \\
\hline $\begin{array}{l}\text { When I put butter or margarine on } \\
\text { bread, I usually spread it thinly }\end{array}$ & $\begin{array}{c}\text { True } \\
\text { False } \\
\text { Not applicable }\end{array}$ & $\begin{array}{l}330(69.3 \%) \\
77(16.2 \%) \\
69(14.5 \%)\end{array}$ & $\begin{array}{c}278(71.3 \%) \\
38(9.7 \%) \\
74(19.0 \%)\end{array}$ & $\begin{array}{l}304(65.1 \%) \\
75(16.1 \%) \\
88(18.8 \%)\end{array}$ & 0.0135 \\
\hline
\end{tabular}


Table 8. Cont.

\begin{tabular}{|c|c|c|c|c|c|}
\hline \multicolumn{2}{|c|}{ Food Preparation Habits Assessed within AFHC * } & $\begin{array}{c}\text { Decreased Physical } \\
\text { Activity during } \\
\text { COVID-19 Pandemic } \\
(n=476)\end{array}$ & $\begin{array}{l}\text { Stable Physical Activity } \\
\text { during COVID-19 } \\
\text { Pandemic } \\
(n=390)\end{array}$ & $\begin{array}{c}\text { Increased Physical } \\
\text { Activity during } \\
\text { COVID-19 Pandemic } \\
(n=467)\end{array}$ & $p^{* *}$ \\
\hline $\begin{array}{l}\text { If I have a packed lunch, I usually } \\
\text { include some chocolate } \\
\text { and/or biscuits }\end{array}$ & $\begin{array}{c}\text { True } \\
\text { False } \\
\text { Not applicable }\end{array}$ & $\begin{array}{l}130(27.3 \%) \\
266(55.9 \%) \\
80(16.8 \%)\end{array}$ & $\begin{array}{l}91(23.3 \%) \\
232(59.5 \%) \\
67(17.2 \%)\end{array}$ & $\begin{array}{l}87(18.6 \%) \\
297(63.6 \%) \\
83(17.8 \%)\end{array}$ & 0.0374 \\
\hline I often have cream on desserts & $\begin{array}{c}\text { True } \\
\text { False } \\
\text { Not applicable }\end{array}$ & $\begin{array}{c}115(24.1 \%) \\
311(65.4 \%) \\
50(10.5 \%)\end{array}$ & $\begin{array}{l}75(19.2 \%) \\
262(67.2 \%) \\
53(13.6 \%)\end{array}$ & $\begin{array}{l}101(21.6 \%) \\
297(63.6 \%) \\
69(14.8 \%)\end{array}$ & 0.1744 \\
\hline
\end{tabular}

* AFHC-Adolescents' Food Habits Checklist [37]; ** chi-square test.

The food consumption habits assessed while using AFHC in the period during the COVID-19 pandemic, as declared by the adolescents from the DAY-19 Study cohort, in subgroups of adolescents declaring decreased, stable, and increased physical activity during the COVID-19 pandemic are presented in Table 9. A higher number of respondents who reported decreased physical activity, when compared with those who reported increased physical activity, declared usually eating a dessert or pudding if there is one available ( $79.0 \%$ vs. $70.4 \%, p<0.0001)$, and often eating sweet snacks between meals ( $55.8 \%$ vs. $43.0 \%$, $p<0.0001)$. At the same time, a lower number of respondents who reported decreased physical activity, when compared with those who reported increased physical activity, declared making sure they eat at least one serving of fruit a day $(80.5 \%$ vs. $89.5 \%$, $p<0.0001)$, avoiding eating lots of sausages and burgers (58.8\% vs. $61.7 \%, p<0.001)$, making sure they eat at least one serving of vegetables or salad a day $(71.6 \%$ vs. $82.4 \%$, $p<0.0001)$, trying to ensure they eat plenty of fruit and vegetables $(73.3 \%$ vs. $87.4 \%$, $p<0.0001)$, often choosing fruit while having a snack between meals $(41.0 \%$ vs. $59.3 \%$, $p<0.0001)$, eating at least three servings of fruit most days ( $45.8 \%$ vs. $66.2 \%, p<0.0001)$, and generally trying to have a healthy diet $(70.8 \%$ vs. $84.6 \%, p<0.0001)$.

Table 9. The food consumption habits assessed while using Adolescents' Food Habits Checklist (AFHC) in the period during the COVID-19 pandemic, as declared by the adolescents from the Diet and Activity of Youth during COVID-19 (DAY-19) Study cohort ( $n=1333$ ) in sub-groups of adolescents declaring decreased, stable, and increased physical activity during the COVID-19 pandemic.

\begin{tabular}{|c|c|c|c|c|c|}
\hline \multicolumn{2}{|c|}{ Food Consumption Habits Assessed within AFHC * } & $\begin{array}{c}\text { Decreased Physical } \\
\text { Activity during } \\
\text { COVID-19 Pandemic } \\
(n=476)\end{array}$ & $\begin{array}{c}\text { Stable Physical Activity } \\
\text { during COVID-19 } \\
\text { Pandemic } \\
(n=390)\end{array}$ & $\begin{array}{c}\text { Increased Physical } \\
\text { Activity during } \\
\text { COVID-19 Pandemic } \\
(n=467)\end{array}$ & $p^{* *}$ \\
\hline $\begin{array}{l}\text { I usually eat a dessert or pudding if } \\
\text { there is one available }\end{array}$ & $\begin{array}{l}\text { True } \\
\text { False }\end{array}$ & $\begin{array}{l}376(79.0 \%) \\
100(21.0 \%)\end{array}$ & $\begin{array}{l}258(66.1 \%) \\
132(33.8 \%)\end{array}$ & $\begin{array}{l}329(70.4 \%) \\
138(29.6 \%)\end{array}$ & $<0.0001$ \\
\hline $\begin{array}{l}\text { I make sure I eat at least one serving } \\
\text { of fruit a day }\end{array}$ & $\begin{array}{l}\text { True } \\
\text { False }\end{array}$ & $\begin{array}{l}383(80.5 \%) \\
93(19.5 \%)\end{array}$ & $\begin{array}{c}350(89.7 \%) \\
40(10.3 \%)\end{array}$ & $\begin{array}{l}418(89.5 \%) \\
49(10.5 \%)\end{array}$ & $<0.0001$ \\
\hline $\begin{array}{l}\text { I avoid eating lots of sausages } \\
\text { and burgers }\end{array}$ & $\begin{array}{c}\text { True } \\
\text { False } \\
\text { Not applicable }\end{array}$ & $\begin{array}{l}280(58.8 \%) \\
142(29.8 \%) \\
54(11.4 \%)\end{array}$ & $\begin{array}{l}241(61.8 \%) \\
72(18.5 \%) \\
77(19.7 \%)\end{array}$ & $\begin{array}{l}288(61.7 \%) \\
100(21.4 \%) \\
79(16.9 \%)\end{array}$ & $<0.0001$ \\
\hline $\begin{array}{l}\text { I make sure I eat at least one serving } \\
\text { of vegetables or salad a day }\end{array}$ & $\begin{array}{l}\text { True } \\
\text { False }\end{array}$ & $\begin{array}{l}341(71.6 \%) \\
135(28.4 \%)\end{array}$ & $\begin{array}{l}319(81.8 \%) \\
71(18.2 \%)\end{array}$ & $\begin{array}{l}385(82.4 \%) \\
82(17.6 \%)\end{array}$ & $<0.0001$ \\
\hline $\begin{array}{l}\text { I try to ensure I eat plenty of fruit } \\
\text { and vegetables }\end{array}$ & $\begin{array}{l}\text { True } \\
\text { False }\end{array}$ & $\begin{array}{l}349(73.3 \%) \\
127(26.7 \%)\end{array}$ & $\begin{array}{l}339(86.9 \%) \\
51(13.1 \%)\end{array}$ & $\begin{array}{l}408(87.4 \%) \\
59(12.6 \%)\end{array}$ & $<0.0001$ \\
\hline $\begin{array}{l}\text { I often eat sweet snacks } \\
\text { between meals }\end{array}$ & $\begin{array}{l}\text { True } \\
\text { False }\end{array}$ & $\begin{array}{l}266(55.8 \%) \\
210(44.2 \%)\end{array}$ & $\begin{array}{l}174(44.6 \%) \\
216(55.4 \%)\end{array}$ & $\begin{array}{l}201(43.0 \%) \\
266(57.0 \%)\end{array}$ & $<0.0001$ \\
\hline $\begin{array}{c}\text { When I have a snack between meals, I } \\
\text { often choose fruit }\end{array}$ & $\begin{array}{c}\text { True } \\
\text { False } \\
\text { Not applicable } \\
\end{array}$ & $\begin{array}{c}195(41.0 \%) \\
223(46.8 \%) \\
58(12.2 \%) \\
\end{array}$ & $\begin{array}{c}214(54.9 \%) \\
131(33.6 \%) \\
45(11.5 \%) \\
\end{array}$ & $\begin{array}{c}277(59.3 \%) \\
135(28.9 \%) \\
55(11.8 \%) \\
\end{array}$ & $<0.0001$ \\
\hline $\begin{array}{l}\text { I eat at least three servings of fruit } \\
\text { most days }\end{array}$ & $\begin{array}{l}\text { True } \\
\text { False }\end{array}$ & $\begin{array}{l}218(45.8 \%) \\
258(54.2 \%)\end{array}$ & $\begin{array}{l}227(58.2 \%) \\
163(41.8 \%)\end{array}$ & $\begin{array}{l}309(66.2 \%) \\
158(33.8 \%)\end{array}$ & $<0.0001$ \\
\hline I generally try to have a healthy diet & $\begin{array}{l}\text { True } \\
\text { False }\end{array}$ & $\begin{array}{l}337(70.8 \%) \\
139(29.2 \%)\end{array}$ & $\begin{array}{l}336(86.2 \%) \\
54(13.8 \%)\end{array}$ & $\begin{array}{l}395(84.6 \%) \\
72(15.4 \%)\end{array}$ & $<0.0001$ \\
\hline
\end{tabular}

* AFHC-Adolescents' Food Habits Checklist [37]; ** chi-square test. 


\subsection{Analysis of the Interfering Factors}

The food purchase habits assessed while using AFHC in the period before the COVID19 pandemic (Supplementary Table S1) and the period during the COVID-19 pandemic (Supplementary Table S2), the food preparation habits assessed in the period before the COVID-19 pandemic (Supplementary Table S3) and the period during the COVID-19 pandemic (Supplementary Table S4), as well as the food consumption habits assessed in the period before the COVID-19 pandemic (Supplementary Table S5) and the period during the COVID-19 pandemic (Supplementary Table S6), stratified by gender, indicated some differences. However, for the majority of habits no gender-dependent differences were stated.

The food purchase habits assessed while using AFHC in the period before the COVID19 pandemic (Supplementary Table S7) and the period during the COVID-19 pandemic (Supplementary Table S8), the food preparation habits assessed in the period before the COVID-19 pandemic (Supplementary Table S9) and the period during the COVID-19 pandemic (Supplementary Table S10), as well as the food consumption habits assessed in the period before the COVID-19 pandemic (Supplementary Table S11) and the period during the COVID-19 pandemic (Supplementary Table S12), stratified by urban/rural environment, indicated some differences. However, for the majority of habits no environment-dependent differences were stated.

\section{Discussion}

Social isolation, caused by the COVID-19 pandemic, induced a lot of changes of everyday life, influencing also the nutrition of children and adolescents. However, based on a review by Stavridou et al. [54], it may be stated that the majority of studies indicated negative changes into less beneficial dietary behaviors. At the same time, in the presented study, it was observed that the majority of changes of food habits in the studied group were positive, as they were associated with reduced fat, sugar, and sweets intake, accompanied by increased consumption of fruit and vegetables. Similarly, in the previous study conducted in Poland, in a population of older adolescents [41], it was noted that during the COVID-19 pandemic, when compared with the period before, a lower number of respondents reported buying pastries, cakes or crisps, and eating a dessert or pudding, as well as a higher number of respondents reported avoiding fried foods, trying to keep their overall sugar intake down, and eating at least one serving of vegetables or salad a day and at least three servings of fruit most days. However, authors of the referred study indicated that similar share of their respondents changed their food habits into more beneficial as into less beneficial ones, and that it was probable that beneficial food habits were forced by the lockdown and the resultant limited possibilities to consume meals in restaurants [41]. Moreover, a recent Polish study revealed also the problem associated with potentially reduced frequency of using catering facilities, influenced not by a need for following a healthy diet, but by the fear of getting sick with COVID-19 [55].

The results of numerous studies indicate a growing problem of the excessive body mass of adolescents during the COVID-19 pandemic $[26,54,56]$, so not only the problem of changing food habits, but also their association with changes of body mass should be reflected. In the presented study, adolescents declaring increase of their body mass during the COVID-19 pandemic were characterized by worse food habits than those who did not declare it. However, especially alarming is that social isolation during this period not only may have induced development of overweight and obesity, but also may have intensified the problem in case of adolescents already being overweight or obese before the pandemic [57]. Some authors indicate that following dietary recommendations during the lockdown was especially hard for excessive body mass children, due to their increase in sedentary lifestyle and increase in feeling hungry [22].

Among various factors influencing food habits of adolescents, there is an important impact of their parents on the food choices of their progeny [58]. In spite of the fact that it was not assessed within the presented study, the family habits may significantly influence 
the analyzed population, so it must be emphasized that the COVID-19 pandemic may have a direct influence on food habits of children and an indirect influence (it may influence habits of parents, and they may consequently influence habits of their progeny). The results of the study by Larson et al. [59] revealed that consuming family dishes may exert a positive influence on the quality of diet and dietary patterns in young adults. This is also confirmed by the results of a study by Merten et al. [60], as they noted that for breakfast consumption, an important determinant is the presence of a parent while consuming this meal. In addition, during the COVID-19 pandemic some researchers noted positive changes of nutrition of families, as they spent more time preparing dishes together $[23,24]$. On the other hand, some authors revealed also negative changes of the nutritional behaviors within families during the COVID-19 pandemic, which were noted especially in the initial phase of the pandemic [61]. In spite of the fact that the influence of family members was not studied in the DAY-19 Study, it cannot be ruled out, taking into account that family members influence one another their food habits, so the negative food habits of adolescents may be shared by all their family members. At the same time, it must also be emphasized that the excessive body mass of children may be associated with excessive body mass of their parents, which may be supposed to be the other serious problem in the period of the COVID-19 pandemic.

The previous studies by other authors indicated that social isolation and prolonging stays at home, commonly associated with internet and social media overuse, are a factors which may have a negative impact on a mental health of children and adolescents in this period, causing anxiety symptoms, depression, and post-traumatic stress [62]. Some studies indicate that both heathy diet [63] and physical activity may positively influence the mental health of children $[64,65]$. The studies conducted in Great Britain, during the COVID-19 pandemic revealed that a higher level of physical activity may counteract the negative effects of COVID-19 fears on mental health and well-being [66]. As in the presented study, the increased physical activity was associated with better food habits, both of them combined may be especially valuable. Moreover, a higher level of physical activity is commonly associated with improved dietary behaviors [67], as it was stated within the DAY-19 Study especially for dietary behaviors associated with fruit and vegetables' consumption. At the same time, Cavadini et al. [33] also noted that adolescents characterized by a higher level of physical activity simultaneously presented a higher frequency of consuming fruit, fruit juices, and salads than in the case of other adolescents.

It should be indicated that increased screen time, which is commonly observed during the COVID-19 pandemic [68], may also be an important factor influencing food habits. A study conducted in a Spanish population of children and adolescents during the COVID-19 pandemic revealed that increased screen time was associated with worse dietary behaviors, including lower intake of fruit, vegetables, fish, pulses, and nuts, but higher intake of fast foods and sweets [69]. Similar observations of increased screen time have also been indicated during the COVID-19 pandemic in the population of Polish adolescents [2]. Taking this into account, it may be supposed that changing food habits which were noted in the conducted study may be also associated with increased screen time and mediated by snacking behaviors [70].

Taking into account the fact that during the period of adolescence some physical activity patterns are developed and strengthened, the observations made in this group during the COVID-19 pandemic may be especially important. The research conducted before the pandemic revealed that adolescents declaring a higher physical activity level at the same time have better nutritional behaviors than the other adolescents [29]. Moreover, during the COVID-19 pandemic the physical activity level in this group has significantly decreased, associated with lack of physical education lessons at school, but also with closed playgrounds and courts $[28,71,72]$ being indicated by adolescents as a reason of their reduced physical activity [27]. This problem may have existed in the studied population and may have caused that only adolescents of the highest motivation had an adequate physical activity level, as they were seeking alternative options to practicing sports. 
As indicated by Barwais [73], during the COVID-19 pandemic physical activity levels decreased, and this was stated for the activities practiced alone, with friends, families, and team sports. Interestingly, a study conducted by Kaur et al. [74] in a population of adults revealed that, especially in the initial phase of the pandemic and resultant lockdowns, respondents had low motivation to practice any sports, while afterwards the situation changed and their motivation increased, as similarly observed in another study for following a healthy diet [61]. This confirms that even during the pandemic, there are possibilities to increase physical activity and to correct improper dietary behaviors, but dedicated tools and adequate time should be applied. At the same time, any digital platforms may be indicated as a promising options, as using them is associated with a higher level of general physical activity during the COVID-19 pandemic and it may motivate users to practice any sport and to develop an individual physical activity routine [75].

In spite of the fact that the conducted study presented some novel observations of the food habits during the COVID-19 pandemic and their association with physical activity and body mass changes in a Polish population of primary school adolescents, some limitations of the study should be listed. The most important issue is associated with the fact that participants were asked about their situation before the COVID-19 pandemic, so gathering retrospective data was included, which may generate so-called recall bias, being a systematic error caused by possible errors of the recollections retrieved by study participants regarding events or experiences from the past. However, it should be indicated that the COVID-19 pandemic was unexpected, so it was not possible to plan an adequate prospective study to conduct such an assessment without recall bias. As such, in spite of the existing bias, the study provided some valuable observations. At the same time, there is also a risk of a self-selection bias, which may have caused that some potential participants of the study did not intend to participate and those who intended had some specific characteristics. The other issue is associated with a fact that participants were not asked about their diet-related diseases diagnosed during the COVID-19 pandemic, and about those diagnosed previously, so some of the observed results may be influenced by specific dietary recommendations which must be followed due to some diseases or disorders. Last but not least, in spite of the fact that some interfering factors were taken into account, there are also other potential factors that were not studied, such as changes of the family socioeconomic status, which may have been caused by the pandemic (as a result of, e.g., unemployment) and also may have influenced the food habits of adolescents.

\section{Conclusions}

In the period of the COVID-19 pandemic and resultant remote education, adolescents in Poland presented different food habits than before. Positive food purchase, preparation, and consumption habits were observed mainly in the sub-groups of adolescents declaring decreased body mass or increased physical activity during the COVID-19 pandemic. It may be suggested that physical activity may support positive changes of dietary behaviors and, when combined positive changes of diet and increased physical activity, they may effectively promote body mass reduction.

Supplementary Materials: The following are available online at https:/ / www.mdpi.com/article/ 10.3390/nu13113711/s1. Supplementary Table S1. The food purchase habits assessed while using Adolescents' Food Habits Checklist (AFHC) in the period before the COVID-19 pandemic, as declared by the adolescents from the Diet and Activity of Youth during COVID-19 (DAY-19) Study cohort $(n=1333)$, stratified by gender. Supplementary Table S2. The food purchase habits assessed while using Adolescents' Food Habits Checklist (AFHC) in the period during the COVID-19 pandemic, as declared by the adolescents from the Diet and Activity of Youth during COVID-19 (DAY-19) Study cohort $(n=1333)$, stratified by gender. Supplementary Table S3. The food preparation habits assessed while using Adolescents' Food Habits Checklist (AFHC) in the period before the COVID-19 pandemic, as declared by the adolescents from the Diet and Activity of Youth during COVID-19 (DAY-19) Study cohort $(n=1333)$, stratified by gender. Supplementary Table S4. The food preparation habits assessed while using Adolescents' Food Habits Checklist (AFHC) in the period during the 
COVID-19 pandemic, as declared by the adolescents from the Diet and Activity of Youth during COVID-19 (DAY-19) Study cohort $(n=1333)$, stratified by gender. Supplementary Table S5. The food consumption habits assessed while using Adolescents' Food Habits Checklist (AFHC) in the period before the COVID-19 pandemic, as declared by the adolescents from the Diet and Activity of Youth during COVID-19 (DAY-19) Study cohort $(n=1333)$, stratified by gender. Supplementary Table S6. The food consumption habits assessed while using Adolescents' Food Habits Checklist (AFHC) in the period during the COVID-19 pandemic, as declared by the adolescents from the Diet and Activity of Youth during COVID-19 (DAY-19) Study cohort $(n=1333)$, stratified by gender. Supplementary Table S7. The food purchase habits assessed while using Adolescents' Food Habits Checklist (AFHC) in the period before the COVID-19 pandemic, as declared by the adolescents from the Diet and Activity of Youth during COVID-19 (DAY-19) Study cohort $(n=1333)$, stratified by urban/rural environment. Supplementary Table S8. The food purchase habits assessed while using Adolescents' Food Habits Checklist (AFHC) in the period during the COVID-19 pandemic, as declared by the adolescents from the Diet and Activity of Youth during COVID-19 (DAY-19) Study cohort ( $n=1333$ ), stratified by urban/rural environment. Supplementary Table S9. The food preparation habits assessed while using Adolescents' Food Habits Checklist (AFHC) in the period before the COVID-19 pandemic, as declared by the adolescents from the Diet and Activity of Youth during COVID-19 (DAY-19) Study cohort ( $n=1333)$, stratified by urban/rural environment. Supplementary Table S10. The food preparation habits assessed while using Adolescents' Food Habits Checklist (AFHC) in the period during the COVID-19 pandemic, as declared by the adolescents from the Diet and Activity of Youth during COVID-19 (DAY-19) Study cohort $(n=1333)$, stratified by urban/rural environment. Supplementary Table S11. The food consumption habits assessed while using Adolescents' Food Habits Checklist (AFHC) in the period before the COVID-19 pandemic, as declared by the adolescents from the Diet and Activity of Youth during COVID-19 (DAY-19) Study cohort ( $n=1333$ ), stratified by urban/rural environment. Supplementary Table S12. The food consumption habits assessed while using Adolescents' Food Habits Checklist (AFHC) in the period during the COVID-19 pandemic, as declared by the adolescents from the Diet and Activity of Youth during COVID-19 (DAY-19) Study cohort $(n=1333)$, stratified by urban/rural environment.

Author Contributions: A.K., D.G. made study conception and design; A.K. performed the research; A.K., D.G. analyzed the data; A.K., D.G. interpreted the data; A.K., D.G. wrote the paper. All authors have read and agreed to the published version of the manuscript.

Funding: This research was funded by the Polish Ministry of Science and Higher Education within funds of Institute of Human Nutrition Sciences, Warsaw University of Life Sciences (WULS), for scientific research.

Institutional Review Board Statement: The study was conducted according to the guidelines of the Declaration of Helsinki, and approved by the Ethics Committee of the Institute of Human Nutrition Sciences, Warsaw University of Life Sciences (WULS-SGGW).

Informed Consent Statement: Informed consent was obtained from all subjects involved in the study.

Conflicts of Interest: The authors declare no conflict of interest.

\section{References}

1. Eckert, K.L.; Loffredo, V.A.; O'Connor, K. Adolescent Physiology. In Behavioral Approaches to Chronic Disease in Adolescence; O’Donohue, W., Ed.; Springer: New York, NY, USA, 2009. [CrossRef]

2. $\quad$ Łuszczki, E.; Sobek, G.; Bartosiewicz, A.; Baran, J.; Weres, A.; Dereń, K.; Mazur, A. Analysis of Fruit and Vegetable Consumption by Children in School Canteens Depending on Selected Sociodemographic Factors. Medicina 2019, 55, 397. [CrossRef] [PubMed]

3. World Health Organization Regional Office for Europe. Health Behaviour in School-Aged Children Study. Available online: https:/ / www.euro.who.int/_data/assets/pdf_file/0006/303477/HBSC-No.7_factsheet_Diet.pdf\%3Fua\%3D1 (accessed on 4 March 2021).

4. Nystrom, A.A.; Schmitz, K.H.; Perry, C.L.; Lytle, L.A.; Neumark-Sztainer, D. The relationship of weight-related perceptions, goals, and behaviors with fruit and vegetable consumption in young adolescents. Prev. Med. 2005, 40, 203-208. [CrossRef] [PubMed]

5. Al Ani, M.F.; Al Subhi, L.K.; Bose, S. Consumption of fruits and vegetables among adolescents: A multi-national comparison of eleven countries in the Eastern Mediterranean Region. BJN 2016, 115, 6. [CrossRef]

6. Lakkakula, A.P.; Zanovec, M.; Silverman, L.; Murphy, E.; Tuuri, G. Black children with high preferences for fruits and vegetables are at less risk of being at risk of overweight or overweight. J. Am. Diet. Assoc. 2008, 108, 1912-1915. [CrossRef] [PubMed] 
7. Matthews, V.L.; Wien, M.; Sabaté, J. The risk of child and adolescent overweight is related to types of food consumed. Nutr. J. 2011, 10, 71. [CrossRef]

8. Mellendick, K.; Shanahan, L.; Wideman, L.; Calkins, S.; Keane, S.; Lovelady, C. Diets rich in fruits and vegetables are associated with lower cardiovascular disease risk in adolescents. Nutrients 2018, 10, 136. [CrossRef] [PubMed]

9. Bondonno, N.P.; Davey, R.J.; Murray, K.; Radavelli-Bagatini, S.; Bondonno, C.P.; Blekkenhorst, L.C.; Sim, M.; Magliano, D.J.; Daly, R.M.; Shaw, J.E.; et al. Associations between fruit intake and risk of diabetes in the AusDiab Cohort. J. Clin. Endocrinol. Metab. 2021, 12, dgab335. [CrossRef]

10. Maynard, M.; Gunnell, D.; Emmett, P.; Frankel, S.; Smith, D.G. Fruit, vegetables, and antioxidants in childhood and risk of adult cancer: The Boyd Orr cohort. J. Epidemiol. Community Health 2003, 57, 218-225. [CrossRef]

11. Farvid, M.S.; Chen, W.Y.; Michels, K.B.; Cho, E.; Willett, W.C.; Eliassen, A.H. Fruit and vegetable consumption in adolescence and early adulthood and risk of breast cancer: Population based cohort study. BMJ 2016, 353, i2343. [CrossRef]

12. Guzek, D.; Głąbska, D.; Groele, B.; Gutkowska, K. Role of fruit and vegetables for the mental health of children: A systematic review. Rocz. Panstw. Zakl. Hig. 2020, 71, 5-13. [CrossRef]

13. Takaoka, Y.; Kawakami, N. Fruit and vegetable consumption in adolescence and health in early adulthood: A longitudinal analysis of the Statistics Canada's National Population Health Survey. BMC Public Health 2013, 13, 1206. [CrossRef]

14. Gerritsen, S.; Renker-Darby, A.; Harré, S.; Rees, D.; Raroa, D.A.; Eickstaedt, M.; Sushil, Z.; Allan, K.; Bartos, A.E.; Waterlander, W.E.; et al. Improving low fruit and vegetable intake in children: Findings from a system dynamics, community group model building study. PLOS ONE 2019, 14, e0221107. [CrossRef]

15. Vereecken, C.; Pedersen, T.P.; Ojala, K.; Krølner, R.; Dzielska, A.; Ahluwalia, N.; Giacchi, M.; Kelly, C. Fruit and vegetable consumption trends among adolescents from 2002 to 2010 in 33 countries. Eur. J. Public Health. 2015, 25 (Suppl. 2), 16-19. [CrossRef]

16. Pedersen, T.P.; Holstein, B.E.; Flachs, E.M.; Rasmussen, M. Meal frequencies in early adolescence predict meal frequencies in late adolescence and early adulthood. BMC Public Health 2013, 13, 445. [CrossRef]

17. Silva, F.A.; Candiá, S.M.; Pequeno, M.S.; Sartorelli, D.S.; Mendes, L.L.; Oliveira, R.M.S.; Netto, M.P.; Cândido, A.P.C. Daily meal frequency and associated variables in children and adolescents. J. Pediatr. 2017, 93, 79-86. [CrossRef] [PubMed]

18. Toschke, A.M.; Küchenhoff, H.; Koletzko, B.; von Kries, R. Meal frequency and childhood obesity. Obes. Res. 2005, 13, 1932-1938. [CrossRef] [PubMed]

19. Ordoñez-Araque, R.; Caicedo-Jaramillo, C.; García-Ulloa, M.; Dueñas-Ricaurte, J. Eating habits and physical activity before and during the health emergency due to COVID-19 in Quito-Ecuador. Hum. Nutr. Metab. 2021, 24, 200122. [CrossRef]

20. Wang, J.; Yeoh, E.K.; Yung, T.K.C.; Wong, M.C.S.; Dong, D.; Chen, X.; Chan, M.K.Y.; Wong, E.L.Y.; Wu, Y.; Guo, Z.; et al. Change in eating habits and physical activities before and during the COVID-19 pandemic in Hong Kong: A cross-sectional study via random telephone survey. J. Int. Soc. Sports Nutr. 2021, 18, 33. [CrossRef] [PubMed]

21. López-Bueno, R.; López-Sánchez, G.F.; Casajús, J.A.; Calatayud, J.; Gil-Salmerón, A.; Grabovac, I.; Tully, M.A.; Smith, L. Healthrelated behaviors among school-aged children and adolescents during the Spanish Covid-19 confinement. Front. Pediatr. 2020, 8, 573. [CrossRef]

22. Nicodemo, M.; Spreghini, M.R.; Manco, M.; Wietrzykowska Sforza, R.; Morino, G. Childhood obesity and COVID-19 lockdown: Remarks on eating habits of patients enrolled in a food-education program. Nutrients 2021, 13, 383. [CrossRef]

23. Teixeira, M.T.; Vitorino, S.R.; da Silva, H.H.; Raposo, L.M.; Aquino, L.A.; Ribas, S.A. Eating habits of children and adolescents during the COVID-19 pandemic: The impact of social isolation. J. Hum. Nutr. Diet. 2021, 11, 12901. [CrossRef]

24. Ruiz-Roso, M.B.; de Carvalho Padilha, P.; Mantilla-Escalante, D.C.; Ulloa, N.; Brun, P.; Acevedo-Correa, D.; Arantes Ferreira Peres, W.; Martorell, M.; Aires, M.T.; de Oliveira Cardoso, L.; et al. Covid-19 confinement and changes of adolescent's dietary trends in Italy, Spain, Chile, Colombia and Brazil. Nutrients 2020, 12, 1807. [CrossRef] [PubMed]

25. Morres, I.D.; Galanis, E.; Hatzigeorgiadis, A.; Androutsos, O.; Theodorakis, Y. Physical activity, sedentariness, eating behaviour and well-being during a COVID-19 lockdown period in Greek adolescents. Nutrients 2021, 13, 1449. [CrossRef]

26. Hourani, H.A.; Alkhatib, B.; Abdullah, M. Impact of COVID-19 lockdown on body weight, eating habits, and physical activity of Jordanian children and adolescents. Disaster Med. Public Health Prep. 2021, 19, 48. [CrossRef]

27. Ng, K.; Cooper, J.; McHale, F.; Clifford, J.; Woods, C. Barriers and facilitators to changes in adolescent physical activity during COVID-19. BMJ Open Sport Exerc. Med. 2020, 23, e000919. [CrossRef] [PubMed]

28. Štveráková, T.; Jačisko, J.; Busch, A.; Šafářová, M.; Kolář, P.; Kobesová, A. The impact of COVID-19 on physical activity of Czech children. PLoS ONE 2021, 16, e0254244. [CrossRef] [PubMed]

29. Schmidt, S.C.E.; Anedda, B.; Burchartz, A.; Eichsteller, A.; Kolb, S.; Nigg, C.; Niessner, C.; Oriwol, D.; Worth, A.; Woll, A. Physical activity and screen time of children and adolescents before and during the COVID-19 lockdown in Germany: A natural experiment. Sci. Rep. 2020, 10, 21780. [CrossRef] [PubMed]

30. Schnaiderman, D.; Bailac, M.; Borak, L.; Comar, H.; Eisner, A.; Ferrari, A.; Giannini, G.; Risso, F.; Vetere, C.; Garibotti, G. Psychological impact of COVID-19 lockdown in children and adolescents from San Carlos de Bariloche, Argentina: Parents' perspective. Arch. Argent. Pediatr. 2021, 119, 170-176. [CrossRef]

31. Perez, D.; Thalken, J.K.; Ughelu, N.E.; Knight, C.J.; Massey, W.V. Nowhere to Go: Parents' Descriptions of Children's Physical Activity During a Global Pandemic. Front Public Health 2021, 9, 642932. [CrossRef] 
32. Eyler, A.A.; Schmidt, L.; Kepper, M.; Mazzucca, S.; Gilbert, A.; Beck, A. Parent Perceptions of Changes in Child Physical Activity During COVID-19 Stay-At-Home Orders. Front Public Health 2021, 9, 637151. [CrossRef]

33. Cavadini, C.; Decarli, B.; Grin, J.; Narring, F.; Michaud, P.A. Food habits and sport activity during adolescence: Differences between athletic and non-athletic teenagers in Switzerland. Eur. J. Clin. Nutr. 2000, 1, S16-S20. [CrossRef]

34. Kiebuła, P.; Tomczyk, K.; Furman, J.; Łabuz-Roszak, B. Association between eating habits and physical activity in primary school student. Wiad. Lek. 2020, 73, 2120-2126. [CrossRef] [PubMed]

35. Kumar, B.; Robinson, R.; Till, S. Physical activity and health in adolescence. Clin. Med. 2015, 15, 267-272. [CrossRef] [PubMed]

36. Badr, H.E.; Lakha, S.F.; Pennefather, P. Differences in physical activity, eating habits and risk of obesity among Kuwaiti adolescent boys and girls: A population-based study. Int. J. Adolesc. Med. Health 2017, 31, 138. [CrossRef]

37. Johnson, F.; Wardle, J.; Griffith, J. The Adolescent Food Habits Checklist: Reliability and validity of a measure of healthy eating behaviour in adolescents. Eur. J. Clin. Nutr. 2002, 56, 644-649. [CrossRef] [PubMed]

38. Proserpio, C.; Pagliarini, E.; Laureati, M.; Frigerio, B.; Lavelli, V. Acceptance of a new food enriched in $\beta$-glucans among adolescents: Effects of food technology neo-phobia and healthy food habits. Foods 2019, 8, 433. [CrossRef]

39. Viggiano, A.; Viggiano, E.; Di Costanzo, A.; Viggiano, A.; Andreozzi, E.; Romano, V.; Rianna, I.; Vicidomini, C.; Gargano, G.; Incarnato, L.; et al. Kaledo, a board game for nutrition education of children and adolescents at school: Cluster randomized controlled trial of healthy lifestyle promotion. Eur. J. Pediatr. 2015, 174, 217-228. [CrossRef]

40. Kalkan, I. The impact of nutrition literacy on the food habits among young adults in Turkey. Nutr. Res. Pract. 2019, 13, 352-357. [CrossRef] [PubMed]

41. Skolmowska, D.; Głąbska, D.; Guzek, D. Differences in Adolescents' Food Habits Checklist (AFHC) scores before and during pandemic in a population-based sample: Polish adolescents' COVID-19 Experience (PLACE-19) Study. Nutrients 2021, 13, 1663. [CrossRef]

42. Skolmowska, D.; Głąbska, D.; Guzek, D. Association between food preferences and food habits in a Polish Adolescents' COVID-19 Experience (PLACE-19) Study. Nutrients 2021, 13, 3003. [CrossRef]

43. Kołota, A.; Głąbska, D. COVID-19 Pandemic and remote education contributes to improved nutritional behaviors and increased screen time in a Polish population-based sample of primary school adolescents: Diet and Activity of Youth during COVID-19 (DAY-19) Study. Nutrients 2021, 13, 1596. [CrossRef]

44. Skolmowska, D.; Głąbska, D.; Guzek, D. Hand hygiene behaviors in a representative sample of Polish adolescents in regions stratified by COVID-19 morbidity and by confounding variables (PLACE-19 Study): Is there any association? Pathogens 2020, 9, 1011. [CrossRef]

45. Guzek, D.; Skolmowska, D.; Głabska, D. Associations between Food Preferences, Food Approach, and Food Avoidance in a Polish Adolescents' COVID-19 Experience (PLACE-19) Study Population. Nutrients 2021, 13, 2427. [CrossRef]

46. Guzek, D.; Skolmowska, D.; Głabska, D. Appetitive traits in a population-based study of Polish adolescents within the PLACE-19 Study: Validation of the adult eating behavior questionnaire. Nutrients 2020, 12, 3889. [CrossRef] [PubMed]

47. The Central Statistical Office in Poland. Available online: https://bdl.stat.gov.pl/BDL/dane/podgrup/temat (accessed on 10 June 2020).

48. The Central Statistical Office in Poland. Available online: https://demografia.stat.gov.pl/BazaDemografia/StartIntro.aspx (accessed on 10 June 2020).

49. Polish Ministry of National Education. Available online: https:/ /rspo.mein.gov.pl/ (accessed on 10 June 2020).

50. Polish Ministry of National Education. Suspension of Classes in Schools. Available online: https://www.gov.pl/web/edukacja/ zawieszenie-zajec-w-szkolach (accessed on 4 March 2021).

51. World Health Organization. Available online: https://www.euro.who.int/en/health-topics/disease-prevention/nutrition/ahealthy-lifestyle/body-mass-index-bmi (accessed on 4 March 2021).

52. Kulaga, Z.; Litwin, M.; Tkaczyk, M.; Różdżyńska, A.; Barwicka, K.; Grajda, A.; Swiader, A.; Gurzkowska, B.; Napieralska, E.; Pan, H. The height-, weight-, and BMI-for-age of Polish school-aged children and adolescents relative to international and local growth references. BMC Public Health 2010, 10, 109. [CrossRef] [PubMed]

53. OLAF Calculator from OLAF Study. Available online: http: / / olaf.czd.pl/index.php?option=com_content\&view=article\&id=103: kalkulator (accessed on 4 March 2021).

54. Stavridou, A.; Kapsali, E.; Panagouli, E.; Thirios, A.; Polychronis, K.; Bacopoulou, F.; Psaltopoulou, T.; Tsolia, T.; Sergentanis, T.N.; Tsitsika, A. Obesity in children and adolescents during COVID-19 pandemic. Child 2021, 8, 135. [CrossRef]

55. Czarniecka-Skubina, E.; Pielak, M.; Sałek, P.; Głuchowski, A.; Kobus-Cisowska, J.; Owczarek, T. Use of food services by consumers in the SARS-CoV-2 pandemic. How the eating habits of consumers changed in view of the new disease risk factors? Nutrients 2021, 13, 2760. [CrossRef]

56. Brooks, C.G.; Spencer, J.R.; Sprafka, J.M.; Roehl, K.A.; Ma, J.; Londhe, A.A.; He, F.; Cheng, A.; Brown, C.A.; Page, J. Pediatric BMI changes during COVID-19 pandemic: An electronic health record-based retrospective cohort study. EClinicalMedicine 2021, 38, 101026. [CrossRef] [PubMed]

57. Nogueira-de-Almeida, C.A.; Del Ciampo, L.A.; Ferraz, I.S.; Del Ciampo, I.R.L.; Contini, A.A.; da V Ued, F. COVID-19 and obesity in childhood and adolescence: A clinical review. J Pediatr. 2020, 96, 546-558. [CrossRef] [PubMed]

58. Neumark-Sztainer, D.; Story, M.; Perry, C.; Casey, M.A. Factors influencing food choices of adolescents: Findings from focus-group discussions with adolescents. J. Am. Diet. Assoc. 1999, 99, 929-937. [CrossRef] 
59. Larson, N.I.; Neumark-Sztainer, D.; Hannan, P.J.; Story, M. Family meals during adolescence are associated with higher diet quality and healthful meal patterns during young adulthood. J. Am. Diet. Assoc. 2007, 107, 1502-1510. [CrossRef] [PubMed]

60. Merten, M.J.; Williams, A.J.; Shriver, L.H. Breakfast Consumption in adolescence and young adulthood: Parental presence, community context, and obesity. J. Am. Diet. Assoc. 2009, 109, 1384-1391. [CrossRef] [PubMed]

61. Hammons, A.J.; Robart, R. Family food environment during the COVID-19 pandemic: A qualitative study. Children 2021, 8, 354. [CrossRef]

62. Guessoum, S.B.; Lachal, J.; Radjack, R.; Carretier, E.; Minassian, S.; Benoit, L.; Moro, M.R. Adolescent psychiatric disorders during the COVID-19 pandemic and lockdown. Psychiatry Res. 2020, 291, 113264. [CrossRef]

63. Głąsska, D.; Kołota, A.; Lachowicz, K.; Skolmowska, D.; Stachoń, M.; Guzek, D. The influence of vitamin D intake and status on mental health in children: A systematic review. Nutrients 2021, 13, 952. [CrossRef]

64. Jassim, G.; Jameel, M.; Brennan, E.; Yusuf, M.; Hasan, N.; Alwatani, Y. Psychological impact of COVID-19, isolation, and quarantine: A cross-sectional study. Neuropsychiatr. Dis. Treat. 2021, 17, 1413-1421. [CrossRef]

65. Okuyama, J.; Seto, S.; Fukuda, Y.; Funakoshi, S.; Amae, S.; Onobe, J.; Izumi, S.; Ito, K.; Imamura, F. Mental health and physical activity among children and adolescents during the COVID-19 pandemic. Tohoku J. Exp. Med. 2021, 253, 203-215. [CrossRef]

66. Wright, L.J.; Williams, S.E.; Veldhuijzen van Zanten, J.J.C.S. Physical activity protects against the negative impact of coronavirus fear on adolescent mental health and well-being during the COVID-19 pandemic. Front Psychol. 2021, 12, 580511. [CrossRef]

67. Doustmohammadian, A.; Keshavarz, S.A.; Doustmohammadian, N.; Ahmadi, N.A. Patterns of physical activity and dietary habit in relation to weight status in adolescent girls. JPS 2013, 4, 2-10.

68. Wagner, B.E.; Folk, A.L.; Hahn, S.L.; Barr-Anderson, D.J.; Larson, N.; Neumark-Sztainer, D. Recreational Screen Time Behaviors during the COVID-19 Pandemic in the U.S.: A Mixed-Methods Study among a Diverse Population-Based Sample of Emerging Adults. Int. J. Environ. Res. Public Health 2021, 18, 4613. [CrossRef]

69. Wärnberg, J.; Pérez-Farinós, N.; Benavente-Marín, J.C.; Gómez, S.F.; Labayen, I.; Zapico, A.G.; Gusi, N.; Aznar, S.; Alcaraz, P.E.; González-Valeiro, M.; et al. Screen Time and Parents' Education Level Are Associated with Poor Adherence to the Mediterranean Diet in Spanish Children and Adolescents: The PASOS Study. J. Clin. Med. 2021, 10, 795. [CrossRef] [PubMed]

70. Nagata, J.M.; Abdel Magid, H.S.; Pettee Gabriel, K. Screen Time for Children and Adolescents During the Coronavirus Disease 2019 Pandemic. Obesity 2020, 28, 1582-1583. [CrossRef]

71. Zenic, N.; Taiar, R.; Gilic, B.; Blazevic, M.; Maric, D.; Pojskic, H.; Sekulic, D. Levels and changes of physical activity in adolescents during the COVID-19 pandemic: Contextualizing urban vs. rural living environment. Appl. Sci. 2020, 10, 3997. [CrossRef]

72. Yomoda, K.; Kurita, S. Influence of social distancing during the COVID-19 pandemic on physical activity in children: A scoping review of the literature. J. Exerc. Sci. Fit. 2021, 19, 195-203. [CrossRef] [PubMed]

73. Barwais, F.A. Physical activity at home during the COVID-19 pandemic in the two most-affected cities in Saudi Arabia. Open Public Health J. 2020, 13, 470-476. [CrossRef]

74. Kaur, H.; Singh, T.; Arya, K.Y.; Mittal, S. Physical fitness and exercise during the COVID-19 pandemic: A qualitative enquiry. Front Psychol. 2020, 11, 590172. [CrossRef] [PubMed]

75. Parker, K.; Uddin, R.; Ridgers, N.D.; Brown, H.; Veitch, J.; Salmon, J.; Timperio, A.; Sahlqvist, S.; Cassar, S.; Toffoletti, K.; et al. The use of digital platforms for adults' and adolescents' physical activity during the COVID-19 pandemic (our life at home): Survey Study. J. Med. Internet Res. 2021, 23, e23389. [CrossRef] 